NASA Technical Memorandum 87816

\title{
Energetics and Dynamics of Simple Impulsive Solar Flares
}

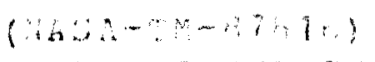

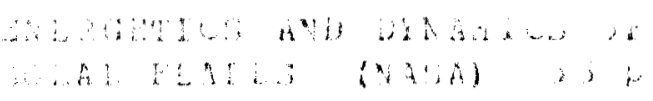 \\ R. Starr, W. A. Heindl, C. J. Crannell, \\ R. J. Thomas, D. A. Batchelor, and A. Magun
}

DECEMBER 1987

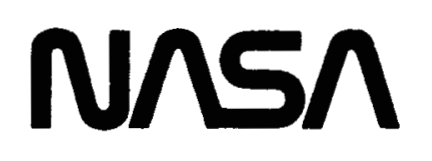




\section{CONTENTS}

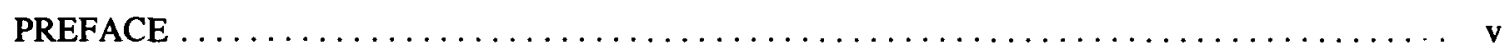

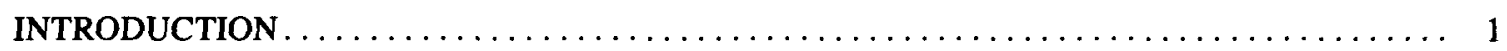

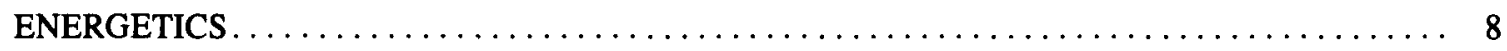

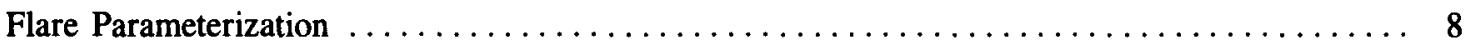

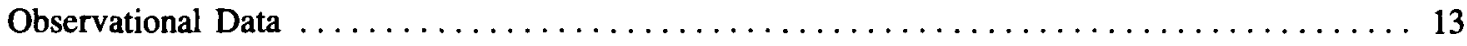

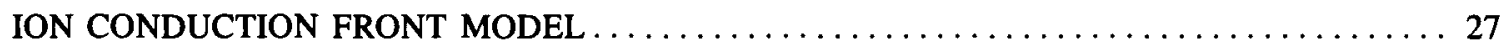

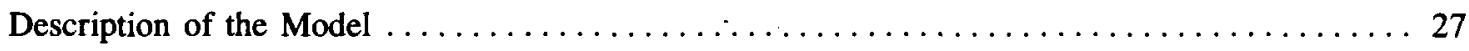

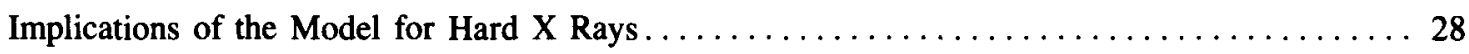

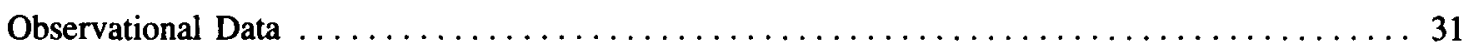

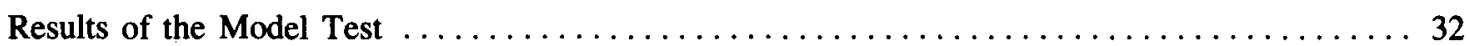

Post Peak Spectral Dynamics and Implications for the Conduction Front Model ........... 39

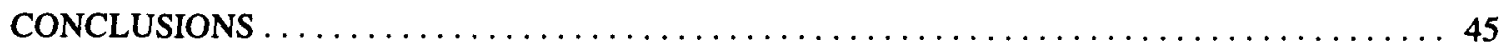

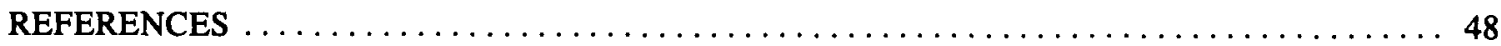




\section{PREFACE}

Many people assisted in the collection, analysis, and presentation of the data in this paper. We thank Brian Dennis, Larry Orwig, Alan Kiplinger, Kim Tolbert, Steve Graham, Bernie Gibson and Shelby Kennard for making available to us the HXRBS data and helping us to analyze and understand what they so generously provided. The GOES data were made available through the National Geophysical Data Center from the Space Environment Laboratories of the National Oceanic and Atmospheric Administration. Assistance with the correlation analysis of the hard X-ray and soft X-ray data was provided by Ed Sullivan, Clara Hughes, Fred Lang, and Andrew Heybey. We are grateful to Werner Neupert for providing significant contributions to our understanding of the relationships between gradual and impulsive emissions. This work was supported in part by NASA RTOP 188-38-01-04 and SMM Guest Investigator Project 370-04-40-02, by NASA grants NSG 5066 and NAG5-774, and by Swiss National Sciences Foundation grant 2.241-0.86SR.

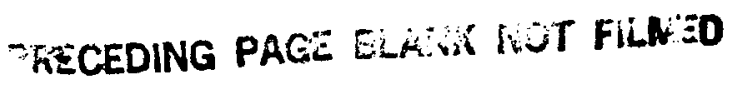




\section{INTRODUCTION}

It is commonly agreed that enhanced soft $\mathrm{X}$-ray emissions during solar flares arise from predominantly thermal processes. The situation with respect to the associated hard $\mathrm{X}$-ray and microwave enhancements, on the other hand, is still the subject of "hot" debate. Hard X rays and microwaves from solar flares were originally interpreted as radiations from supra-thermal accelerated electrons (e. g. Peterson and Winkler 1959; Anderson and Winkler 1962). Chubb, Kreplin, and Friedman (1966) pointed out that the hard X-ray observations also could be accounted for by thermal plasmas of temperature $\mathrm{T} \geq 10^{8} \mathrm{~K}$. Kahler (1971, 1975) and others rejected the thermal explanation on the grounds that heat conduction would cool such plasmas too rapidly to account for observed source lifetimes, unless implausible quantities of energy were expended to sustain the temperature. But then groups such as Brown, Melrose and Spicer (1979) developed thermal models of hard X-ray production that avoided the explicit problems noted by Kahler. Subsequently, many studies of hard X-ray bursts were carried out from both the thermal and the nonthermal points of view. The following is a brief description of a representative sample.

Crannell et al. (1978) analyzed hard X-ray and microwave observations of 22 simple impulsive bursts, using a thermal interpretation and the assumption of a single electron distribution, and discovered a number of suggestive correlations of parameters such as rise time with source size, and rise time with fall time. Matzler et al. (1978) showed that two of the flares considered by Crannell et al. (1978) exhibited the relationship between dynamics of the emission measure and 
the temperature expected for a reversible process such as an adiabatic compression and expansion of the source. Brown, Craig, and Karpen (1980) showed that the Brown, Melrose, and Spicer (1979) model predicted an emission measure-temperature relationship unlike that observed by Matzler et al.; however, an ensemble of small energy releases, each with dynamical behavior predicted by the Brown, Melrose, and Spicer model, could reproduce the observed emission measuretemperature evolution if the energy releases follow the temporal profile derived by Brown, Craig, and Karpen. Wiehl, Schochlin, and Magun (1980) analyzed the dynamic microwave spectrum of nine impulsive events and interpreted the temporal dispersion that they found as evidence for the Brown, Melrose, Spicer model.

Observations of impulsive flares with the Hard X-ray Imaging Spectrometer (HXIS) on the Solar Maximum Mission (SMM) were initially believed to favor the thick-target nonthermal model over a thermal interpretation, because of the appearance of footpoints in a few of the 16-30 keV X-ray images (cf. Duijveman, Hoyng, and Machado 1982). A detailed quantitative analysis of the image sequences by Mackinnon, Brown, and Hayward (1985), however, demonstrated that the image observations with HXIS were equally consistent with a thermal interpretation. Because the hard $\mathrm{X}$-ray emission is optically thin, line-of-sight effects make footpoints of loops appear more intense when the loops are viewed near the center of the solar disk. Mackinnon, Brown and Hayward also showed that initial claims of simultaneous X-ray emission from widely separated footpoints did not have conclusive quantitative support, so the role of electron beams remains unclear. 
Brown et al. (1983) attempted to explain the vertical structure of five flares as determined from observations of each flare with two spacecraft, one having a view of the whole flare region while the view from the other spacecraft was partially occulted by the solar limb. While the thick-target electron beam model could account for the spatial and spectral observations of the flares at any one time, the model was inconsistent with the temporal evolution of flare structures, even if allowance was made for enhanced coronal densities due to "chromospheric evaporation."

Smith and Brown (1980), considering a thermal model of impulsive hard X-ray and microwave emission, claimed that the electrons in the high-energy tail of a Maxwellian distribution would escape from a flaring loop, and thus could not produce the observed microwave emission. This conclusion was challenged by Batchelor et al. (1985), however, because the arguments of Smith and Brown (1980) were based on consideration of only one-dimensional motion of the high-energy electrons; a three-dimensional treatment of the high-energy tail showed that electrons in the tail with pitch angles of $\sim 45$ degrees or more would not escape, and could account for the microwave emission.

As can be seen from the variety of results and conclusions described above, the issue of thermal versus nonthermal production of impulsive flare radiations has yet to be settled.

The present work is a study of flare energetics and dynamics using 
observations of simple impulsive spike bursts. A large, homogeneous set of events was selected to enable the most definitive tests possible of competing flare models, in the absence of spatially resolved observations. Throughout this effort, special emphasis was given to distinguishing between thermal and nonthermal models. Previous studies of the energetics of impulsive versus gradual flare emissions have led to inconclusive results. For example, Crannell, Karpen, and Thomas (1982, hereinafter CKT) showed that good correlations existed between the energetics of gradual and impulsive emission sources when the latter were interpreted as thermal, but comparable correlations also were found from another set of flares analyzed with the assumption that the hard $X$ rays were due to thick-target impact of supra-thermal electron beams, as reported in Wu et al. (1986). With yet a third data set analyzed for the SMM Workshop, soft X-ray peak counting rates and total hard X-ray counts also were found to yield comparable correlations without any model assumptions.

A number of circumstances may have contributed to the failure of these studies to distinguish between the two major classes of flare theories. Of the studies just cited, only the thermal model test was carried out with a homogeneous set of events. Furthermore, each of the data sets used was different in size, so that intercomparison of results was somewhat ambiguous. These difficulties have prompted us to repeat and extend the CKT study with a much larger number of homogeneous events (48 versus 20), and to consider energetics relationships within this single, well-chosen set between soft $X$-ray parameters and all three types of hard X-ray and microwave parameters, i. e., those derived from 
a thermal model, those from a nonthermal model, and those that are model independent.

In addition, a more detailed analysis was carried out for a smaller set of simple impulsive spike bursts. These six events were selected because (1) they were big enough to have sufficient photon statistics in the hard $X$-ray domain to allow spectral fits to be performed at onesecond time intervals and (2) they produced sufficient microwave emission above the threshold of detection at the Bern Radio Observatory to enable coincident hard $\mathrm{X}$-ray and microwave analysis to be carried out throughout the event. In this analysis, several flare parameters were examined, including temperature and emission measure, as a function of time during the rise and fall of each spike burst.

With coincident hard $\mathrm{X}$-ray and optically thick microwave emission throughout the rise of these six flares, it was possible to do a detailed test of the thermal ion conduction front flare model of Batchelor et al. (1985). Batchelor et al. suggested that a collisionless conduction front moves along a flaring loop at the ion-sound speed and that the rise time of a flare is approximately equal to the loop length divided by the ion sound speed. This model was tested in the present work by comparison of the corresponding hard X-ray parameters determined throughout the rise of each event.

The particular strength of this investigation is that it utilized homogeneous, high-quality observations of a relatively large, select set of simple impulsive spike bursts that were analyzed in a uniform, 
systematic manner from both the thermal and the nonthermal points of view. From the choice of impulsive events with only a single rise and fall, it is much more likely that the resulting hard $\mathrm{X}$-ray and microwave emissions arise from just one spatial site. Caution must be exercised on this point, nevertheless, as is demonstrated by the microwave observations recently reported by Gary and Hurford (1986), showing two distinct spatial components that rose and fell in intensity with the temporal characteristics of one simple spike burst. The present analysis is based on the assumption that the two emissions, hard X-ray and microwave, do arise from a common electron population, so that information from both can be combined to characterize the (one) impulsive source, that can then be directly and unambiguously compared to the (assumed single) source of the event's gradual soft X-ray emission. If there were any significant difference between the abilities of thermal and nonthermal models to estimate the energy of the energetic electrons relative to the energy associated with the soft $X$-ray emitting plasma, this highly select set of simple flares would offer an optimal opportunity for revealing it.

The hard $\mathrm{X}$-ray data used in this investigation were obtained with the Hard X-Ray Burst Spectrometer (HXRBS) on the Solar Maximum Mission (SMM) spacecraft. The HXRBS measurements covered the spectral range between 26 and $461 \mathrm{keV}$, which includes all radiations that are energetically important to the impulsive phase. The measurements were made with a sensitivity and temporal resolution that enable meaningful spectral fits to be performed on time scales as short as $1 \mathrm{~s}$ at the peak of all events in our study and during the evolution 
of the six events selected for more detailed analysis. For the latter events, microwave observations at a number of fixed frequencies, with timing accuracy and resolution comparable to those of HXRBS, were provided by the Institute for Applied Physics of the University of Bern (IAPB). Values of the associated soft X-ray fluxes were provided by the National Oceanic and Atmospheric Administration (NOAA) from measurements obtained with instruments on board their series of Geostationary Operational Environmental Satellites (GOES). The GOES data consisted of full-Sun fluxes in two broad-band channels (1.5 to 12 and 3 to $25 \mathrm{keV}$ ), which allowed the gradual component of each event to be characterized with a temporal resolution of $3 \mathrm{~s}$.

In the next Section, we describe the emission mechanisms and specific flare models that were considered in this investigation, and present derivations of the parameters that were tested. The data that form the basis of the present analysis are discussed in Section II.B. together with indications of what measurements were used, how events were selected, and how parameters to be tested were calculated from the raw data. Results of the correlation analysis between soft X-ray and hard $\mathrm{X}$-ray energetics parameters are presented in Section II.C. In Section III. the ion conduction front model and tests of that model with the well-observed spike bursts are described. Finally, conclusions drawn from this investigation and suggestions for future studies are discussed in Section IV. 


\section{ENERGETICS}

\section{A. FLARE PARAMETERIZATION}

1. Soft X-Ray Thermal Emission

Because the soft $\mathrm{X}$-ray (SXR) emission in a solar flare is produced predominantly by non-relativistic electron bremsstrahlung, the thermal energy content of the source plasma can be approximated by:

$$
U_{\text {therm }}[S X R]=\int N(T) k d T \text {, }
$$

where $k$ is Boltzmann's constant and $N(T)$ is the total number of electrons with a Maxwellian velocity distribution characterized by the temperature parameter T. Although such sources are known to be multithermal, their energy content can be expressed in terms of an effective temperature so that:

$$
U_{\text {therm }}[S X R]=N k T_{\text {eff }}=(n V\rangle k T_{\text {eff }} \text {, }
$$

where $\mathbf{n}$ is the electron density and $\mathrm{V}$ is the emitting source volume. Unfortunately, measurements required to make independent estimates of the source size or density are not in general available for the flares in our study. Broad-band soft X-ray observations provide only effective temperatures and emission measures, which can be defined for isothermal sources as:

$$
\operatorname{EM}[S X R]=n^{2} V \square n(n V)
$$

Consequently, the total energy in the soft X-ray source cannot be 
determined directly. However, combining the above expressions leads to:

$$
k T_{\text {eff }} \operatorname{EM}[S X R]=n \cdot U_{\text {therm }}[S X R] \text {. }
$$

Thus, for purposes of the present analysis, the energetics of the SXR emitting source are characterized by the product of Boltzmann's constant, its effective temperature, and its emission measure, which is then approximately equal to the total thermal energy times the electron density of the source.

\section{Hard X-Ray Thermal Emission}

Our treatment of the thermal energy in a hard X-ray (HXR) emitting source at the burst's peak is similar to that described above for the SXR case, but with a few modifications. Here, the isothermal emission measure is defined as:

$$
\mathrm{EM}[\mathrm{HXR}]=\mathrm{n}^{2} \mathrm{~V}=(\mathrm{n} \mathrm{V})^{2} / \mathrm{V} \text {, }
$$

so that the thermal energy content of the flaring HXR source can be expressed as:

$$
U_{\text {therm }}[H X R]=(n V) k T_{e f f}=(E M[H X R] V)^{1 / 2} k T_{e f f} \cdot
$$

For the HXR source, the emitting volume is estimated from a length (L) determined from the plasma's ion sound speed $\left(c_{s}\right)$ and the measured rise time of the impulsive burst $\left(t_{r}\right)$ using the approach of Batchelor et al. (1985): 


$$
\begin{gathered}
V=L^{3}, \\
L=c_{s} t_{r}, \\
c_{s}=\left(k T_{e} / m_{i}\right)^{1 / 2},
\end{gathered}
$$

where $m_{i}$ is the average ion mass, taken to be the proton mass. Assuming that $T_{e f f}$ and $T_{e}$ are the same, these expressions can be combined to give:

$$
U_{\text {therm }}[H X R]=\operatorname{EM}[H X R]^{1 / 2}\left(k T_{e f f}\right)^{7 / 4} t_{r}{ }^{3 / 2} m_{i}^{-3 / 4} \text {. }
$$

This involves an overly simplified view of the burst geometry that contributes as much as a factor of two to the $\mathrm{rms}$ scatter in the calculated energy content of the HXR source (cf. Batchelor et al. 1985).

\section{Hard X-Ray Nonthermal Emission}

It was first shown by Brown (1971) that hard $\mathrm{X}$-ray burst emission with a power-law spectrum can be produced by a beam of electrons whose energy distribution is itself in the form of a power law (non-Maxwellian and therefore "nonthermal"). But, even then, the energy lost by the beam electrons depends on the nature of the target that they encounter when producing the observed bremsstrahlung $X$ rays. Two extreme possibilities are the thin-target and thick-target cases. In the thin-target case, the beam electrons pass rapidly through the target region, losing a negligible amount of energy during the brief encounter. The efficiency of bremsstrahlung emission is directly proportional to the column density of the target, because each beam 
electron has a very small probability of producing an $X$ ray in the so-called "thin target" region. In the thick-target case, on the other hand, the target region is assumed to present such a large column density to the beam electrons that they lose all of their energy in the target, mainly by Coulomb collisions with the ambient electrons of the plasma. Even in this case, however, the beam electrons convert only a very small fraction $\left(\sim 10^{-5}\right)$ of their initial energy into radiation, while the majority goes into heating of the ambient plasma. For X-ray production by nonthermal electron beams in the present study, only the thick-target mechanism is considered because the thin target is too inefficient to be consistent with the total energy observed in hard X-ray emission (Brown 1975).

In either case, if the observed X-ray photon spectrum is in the form of a power law, it can be written as:

$$
\frac{\mathrm{dN}\left(E_{p}\right)}{d E_{p}}=A 1\left(E_{p} / E_{m}\right)^{-A 2} \text { photons } \mathrm{cm}^{-2} \mathrm{~s}^{-1} \mathrm{keV}^{-1}
$$

where $E_{p}$ is the photon energy in $k e V$, and $E_{m}$ is a "mean" energy also in keV. In our data reduction procedure, we have taken the value of $E_{m}$ to be $50 \mathrm{keV} . \quad E_{m}$ is used to make $A 1$ and $A 2$ as nearly independent of each other as possible in the least squares fitting procedure. A1 and A2 then become the defining parameters for each X-ray photon spectrum. The rate at which electrons of various energies from the exciting beam enter into the X-ray emitting target region can be expressed in a similar form: 


$$
I\left(E_{e}\right)=B 1 E_{e}^{-B 2} \text { electrons } s^{-1} \mathrm{keV}^{-1}
$$

where $E_{e}$ is the electron energy in keV. As described in the SMM Workshop Report (Wu et al. 1986) for the case of "thick target" emission, the electron beam spectral parameters can be found from those defining the observed $\mathrm{X}$-ray photon spectrum by means of the following relationships:

$$
\mathrm{B} 2=\mathrm{A} 2+1 \text {, }
$$

and

$$
\mathrm{B} 1=3 \times 10^{33} \mathrm{~A} 1 \mathrm{E}_{\mathrm{m}}^{\mathrm{A} 2} \mathrm{~A} 2(\mathrm{~A} 2-1)^{2} \mathrm{~b}\left(\mathrm{~A} 2-\frac{1}{2}, \frac{3}{2}\right),
$$

where $b(m, n)$ is the beta function. The time-dependent flux of energy into the target due to electrons with kinetic energies above a threshold energy $E_{0}$ can thus be expressed:

or

$$
P\left(E_{0}\right)=\int_{E_{0}}^{\infty} E_{e} I\left(E_{e}\right) d E_{e} k e V s^{-1},
$$

$$
P\left(E_{0}\right)=3 \times 10^{33} \mathrm{~A} 1 \mathrm{E}_{m}^{\mathrm{A} 2} \mathrm{~A} 2(\mathrm{~A} 2-1) \mathrm{b}\left(\mathrm{A} 2-y_{2}, y_{2}\right) \mathrm{E}_{0}^{-\mathrm{A} 2+1} \mathrm{keV} \mathrm{s}^{-1},
$$

or

$$
P\left(E_{0}\right)=5 \times 10^{24} \mathrm{~A} 1 \mathrm{E}_{m}^{\mathrm{A} 2} \mathrm{~A} 2(\mathrm{~A} 2-1) \mathrm{b}\left(\mathrm{A} 2-\frac{1}{2}, y_{2}\right) E_{0}^{-\mathrm{A} 2+1} \mathrm{erg} \mathrm{s}{ }^{-1} .
$$

Choosing $E_{0}=25 \mathrm{keV}$ gives the value of $P_{25}$ used by Hoyng et al. (1976), which is the energy per second entering the $\mathrm{X}$-ray emitting target region in the form of nonthermal electrons with energies above 25 
keV. The total energy in the electron beam can, therefore, be calculated by integrating over the duration of the event, so that:

$$
U_{\text {beam }}[H X R]=\int_{\text {Preflare }}^{\text {Postflare }} \text { P }_{\text {ps d }} \text { erg },
$$

or

$$
U_{\text {beam }}[H X R]=\sum_{i}\left(P_{25}\right)_{i} \Delta t \text { erg },
$$

where $\Delta t$ is the length of time over which data are accumulated for each individual determination of the HXR spectral fit during the flare.

\section{B. OBSERVATIONAL DATA}

To identify a large, homogeneous set of hard X-ray measurements of simple, impulsive solar flares, a search was made through all observations obtained with the SMM/HXRBS instrument during the interval February 1980 to December 1982. From that search, an initial list of 118 simple impulsive spike bursts was compiled. The list comprised all of the hard X-ray events that exhibited a temporal profile at the full resolution of $128 \mathrm{~ms}$ of a single rise and fall to within 10 percent of the peak flux, with clean observations throughout the event, uncontaminated by data dropouts, particle interference, satellite night, or overlap with other flares. Of these, 48 events were found that had sufficient counting rates for determining the required hard X-ray spectral parameters based on both thermal and nonthermal emission mechanisms and, in addition, that had GOES observations of adequate quality to determine the effective temperature and emission measure of the associated soft X-ray emitting plasma. This group of 48 carefully selected events makes up the master set used in the energetics portion 
of our study.

The spectral analysis procedure for the HXRBS data was applied only to measurements in Channel 2 or above, in order to avoid instrumental effects that make the lowest energy channel unreliable. Hereafter, the phrase "all channels" will be used to mean all except Channel 1 . In addition, background levels as derived from linear fits to pre- and post-burst measurements were systematically subtracted out prior to any subsequent analysis, so that results would refer only to flare enhanced radiations. These data, summed over all channels at their full temporal resolution of $128 \mathrm{~ms}$, were used to find the hard X-ray peak time, and the hard $\mathrm{X}$-ray rise time of each burst. Rise time is defined here as twice the length of time taken by the burst to increase from half of its peak intensity to its peak intensity. Also, for each event, the peak hard X-ray emission rate, and the total flare hard X-ray emission integrated over the whole flare were taken from the HXRBS Event Listing (Dennis et al. 1985). The flare enhanced peak emission was determined by subtracting an average background of 40 counts $\mathbf{s}^{-1}$ from the counting rates tabulated in this reference. Total emission is simply the sum of all enhanced HXRBS counts over the duration of the event.

Two types of spectral fitting procedures were then employed for each event, one using a thermal bremsstrahlung function and the other using a power law. The data for this part of the analysis were summed over time intervals of sufficient duration to obtain adequate statistical significance for these fits while retaining adequate temporal resolution and consistent treatment for each event. The spectral parameters were 
obtained by an iterative forward convolution procedure (Batchelor 1984).

Each thermal fit was made at the peak of the HXR burst, and resulted in an effective temperature and emission measure ( $T_{\text {eff }}[\mathrm{HXR}$ ] and EM[HXR]) which we used to characterize the event. The spectral form to which the data were fitted is:

$$
\frac{d N\left(E_{p}\right)}{d E_{p}}=C 1 E_{p}^{-1.4} \exp \left[-\left(E_{p}-E_{m}\right) / C 2\right] \text { photons } \mathrm{cm}^{-2} \mathrm{~s}^{-1} \mathrm{keV}^{-1}
$$

$E_{p}$ is the photon energy in $k e V$, and $E_{m}$ is an approximate "mean" energy set to $50 \mathrm{keV}$ for the purposes of the present analysis. The constants $\mathrm{C} 1$ and $\mathrm{C} 2$ result from several iterations of a standard least-squares fitting routine to the observed count-rate distribution (Bevington 1969). The desired values of effective temperature and emission measure for the thermal case are then related to these spectral fit parameters as follows:

$$
T_{\text {eff }}[\mathrm{HXR}]=\mathrm{C} 2 / \mathrm{k} \text { Kelvin, }
$$

and

$$
\mathrm{EM}[H \mathrm{HXR}] \square \mathrm{C} 1 \mathrm{C} 2^{0.1} \exp \left[\mathrm{E}_{m} / \mathrm{C} 2\right] 10^{45} /\left(1.3 \times 10^{3}\right) \mathrm{cm}^{-3}
$$

where $\mathrm{k}$ is Boltzmann's constant equal to $8.617 \times 10^{-8} \mathrm{keV} \mathrm{deg}^{-1}$.

The power-law fits were obtained at 2-8 intervals throughout each event, and were used to infer instantaneous energies in a nonthermal electron beam under the assumption that such a beam was producing the observed hard X-ray emission by thick-target interactions, as described 
above in Section II.A.3. These instantaneous energies were then summed over the duration of the burst to give an estimate of the total beam energy ( $\left.U_{\text {beam }}[H X R]\right)$ that would have been required. Further details on the spectral analysis procedures together with a brief description of the physical assumptions regarding the thick-target, nonthermal beam model can be found in Batchelor (1984) and Wu et al. (1986).

For the soft X-ray component of each event, the effective color temperature and corresponding emission measure were estimated from GOES observations by the ratio technique described in Thomas, Starr, and Crannell (1985). In this procedure, the effective color temperature is defined to be the temperature of an isothermal plasma which would produce the observed ratio of responses in the two GOES detectors. The value of $T[S X R]$ and EM[SXR] listed in Table 1 were derived for the 3-s interval midway between the times of the maximum intensity in the two GOES channels. (The higher energy channel typically peaks several seconds before the lower energy channel.) The peak flux value listed was the sum of the highest rates recorded in each channel.

\section{RESULTS}

In Table 1, values are listed for each of the parameters that were used to characterize the energetics of the 48 simple, impulsive solar flares. For the hard X-ray component, these include the HXRBS event number, the date and time of burst maximum, the rise time $\left(t_{r}\right)$, the peak count rate, the total number of detected counts, the effective temperature and emission measure from the thermal fits and the photon spectral index from the power-law fits. The corresponding soft X-ray 


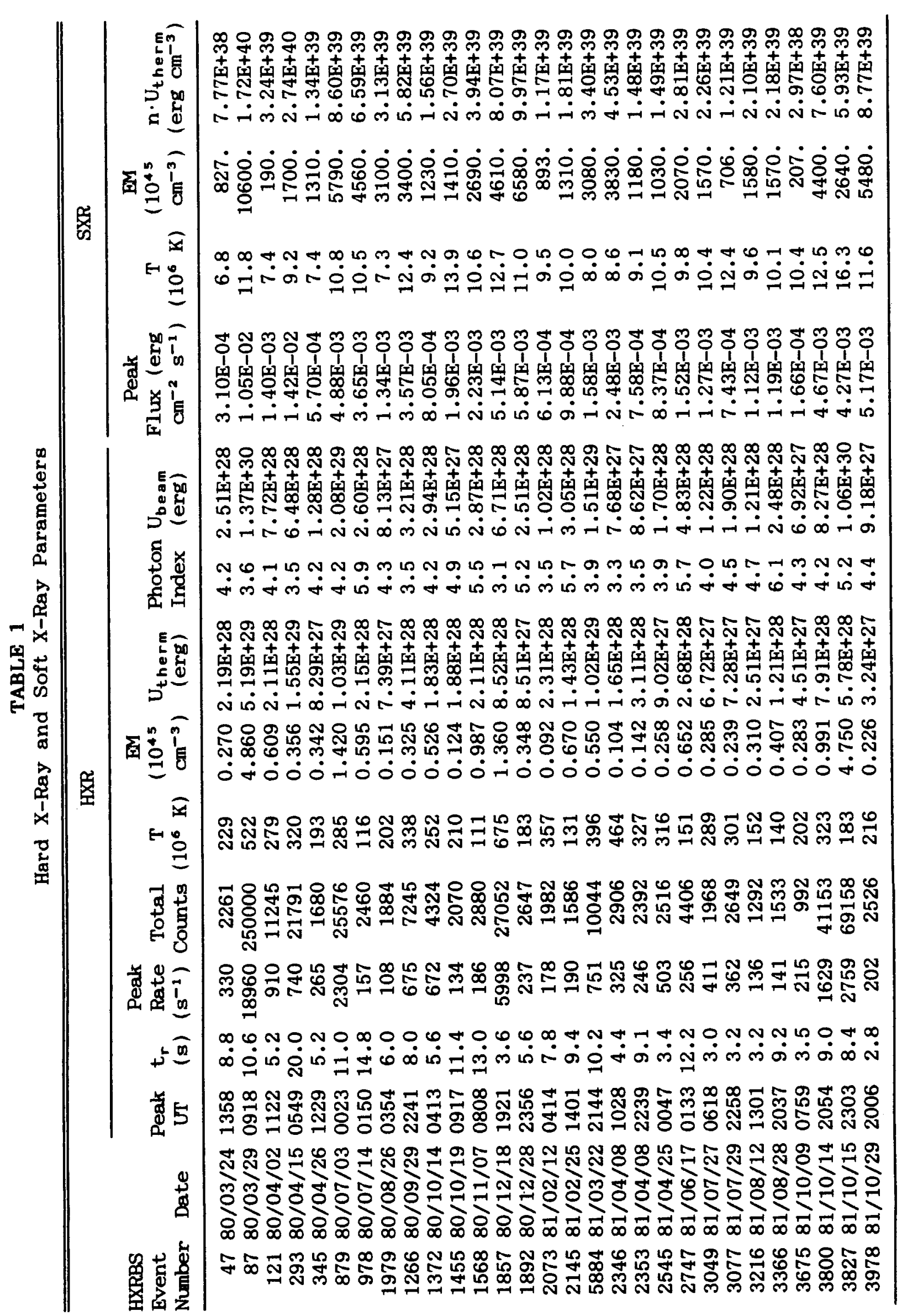




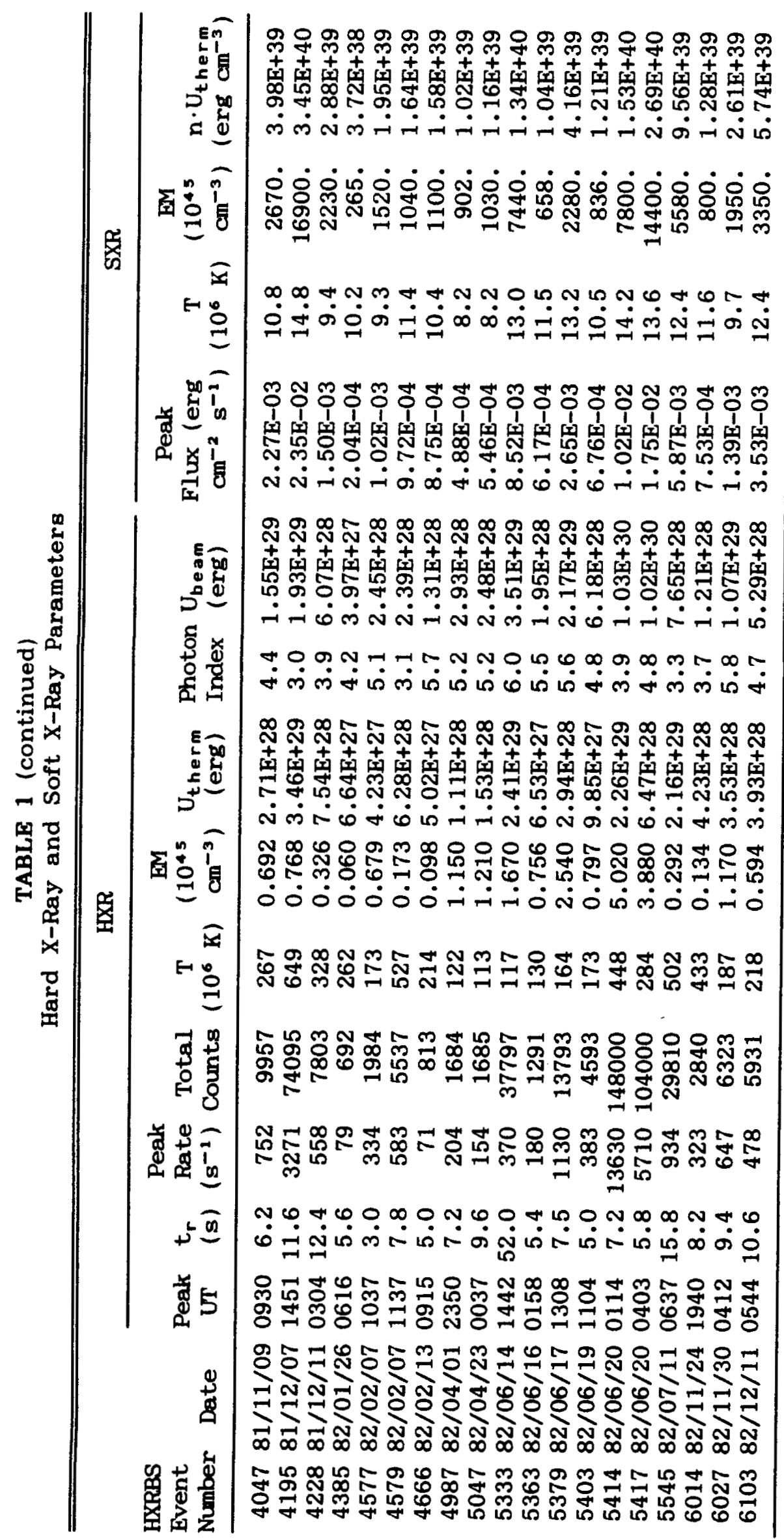


event is described by the peak flux and effective temperature and emission measure. In addition, values are given for the energy content of the energetic electron populations responsible for the observed hard $\mathrm{X}$ rays under the separate assumptions of thermal and thick-target, nonthermal emission mechanisms. The product of the thermal energy of the soft X-ray emitting plasma times its density is also presented.

Initially, correlation analyses were carried out on an event by event basis for the logarithms of four different pairs of energetics parameters for comparison with earlier work (CKT; Wu et al. 1986). These include $n \cdot U_{\text {therm }}[S X R]$ versus $U_{\text {beam }}[H X R]$, $n \cdot U_{\text {therm }}[S X R]$ versus $\mathrm{U}_{\text {therm }}[\mathrm{HXR}]$, Peak Flux[SXR] versus Total Counts[HXR], and Peak Flux[SXR] versus Peak Rate[HXR]. The scatter plots for each of these cases are shown in Figures 1 through 4, which also indicate the regression lines that best fit the data. Subsequently, correlation analyses were determined for logarithms of five additional energetics parameter pairs: $n \cdot U_{t h e r m}[S X R]$ versus Total Counts[HXR], Peak Flux[SXR] versus $U_{\text {beam }}[H X R]$, Peak Flux[SXR] versus $U_{\text {therm }}[H X R]$, $n \cdot U_{\text {therm }}[S X R]$ versus Peak Rate[HXR], and $n \cdot U_{\text {therm }}[S X R]$ versus $U_{\text {therm }} \cdot\left[\left\langle c_{s}\right\rangle / c_{s}\right]^{3 / 2}[H X R]$. Values for each correlation coefficient (r) and best-fit two-dimensional regression line (in the form $y=a+b x$ ) are given in Table 2, along with two measures of the strength of each relationship, namely: $\mathrm{P}_{c}(\mathrm{r}, \mathrm{N})$, the probability that the derived correlation coefficient or a greater one would be found from two uncorrelated distributions, where $\mathrm{N}$ is the total number of parameter pairs; and a statistic that is the root mean square (rms) perpendicular deviation of the parameter values from the best-fit regression line. This best fit 


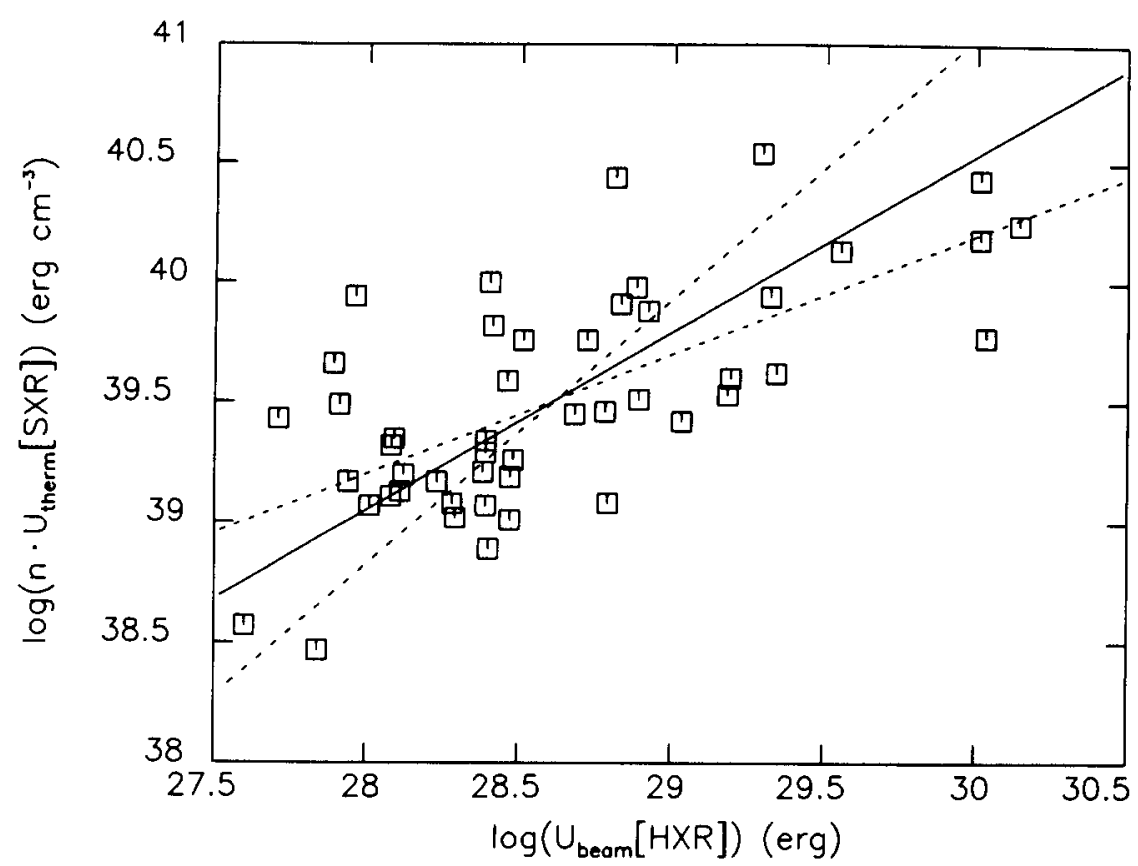

Figure 1. Log of the product of the soft X-ray thermal energy $\left(\mathrm{U}_{\mathrm{therm}}\right)$ and the electron density $(\mathrm{n})$ plotted versus the $\log$ of $U_{\text {beam }}[H X R]$ for the sample of 48 events. The solid line is the linear representation of the correlation that minimizes the perpendicular deviations. The dashed line, with the shallower slope, is the linear representation that minimizes the rms deviations with respect to the values of the ordinate, $\log$ $\mathrm{n} \cdot \mathrm{U}_{\text {therm }}[\mathrm{SXR}]$; while the dashed line with the steeper slope minimizes the deviations with respect to the abscissa, $\log \mathrm{U}_{\text {beam }}[\mathrm{HXR}]$.

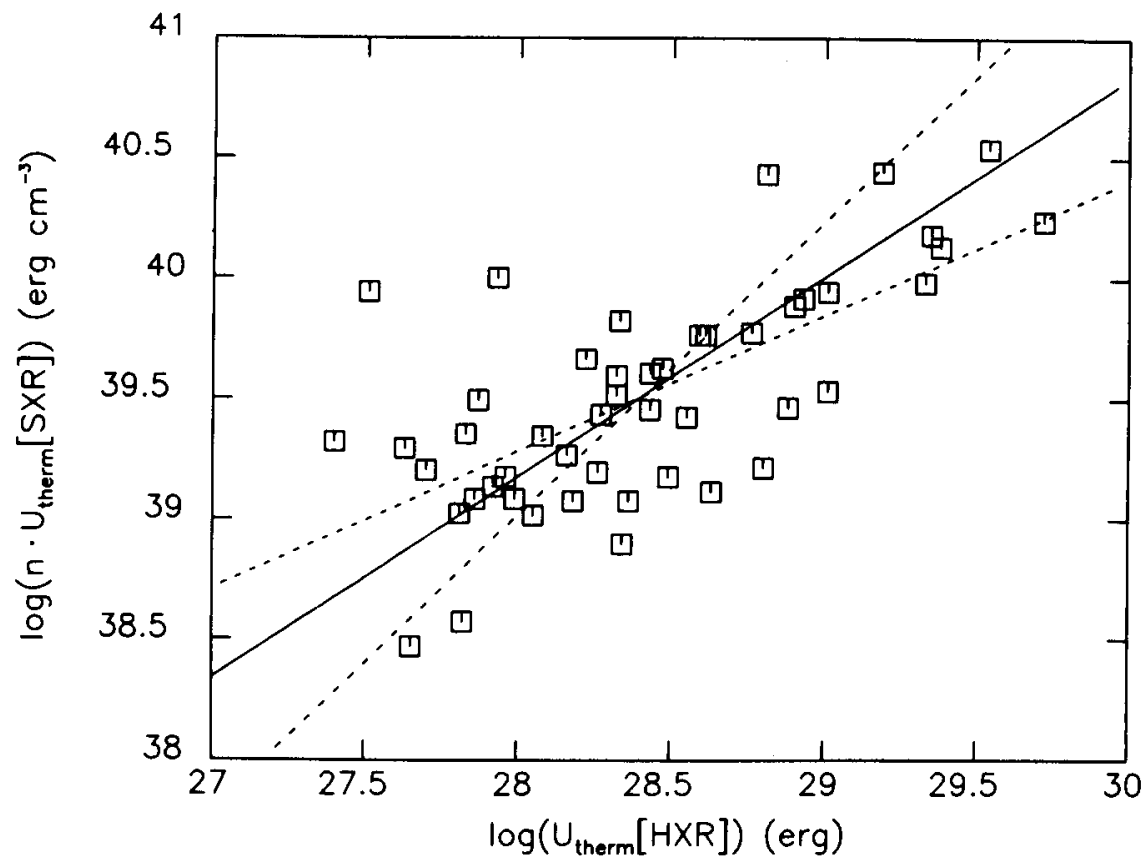

Figure 2. Log of the product of the soft X-ray thermal energy $\left(\mathrm{U}_{\text {therm }}\right)$ and the electron density (n) versus the $\log$ of $U_{\text {therm }}[H X R]$. The best fit lines are as described in the caption to Figure 1 . 


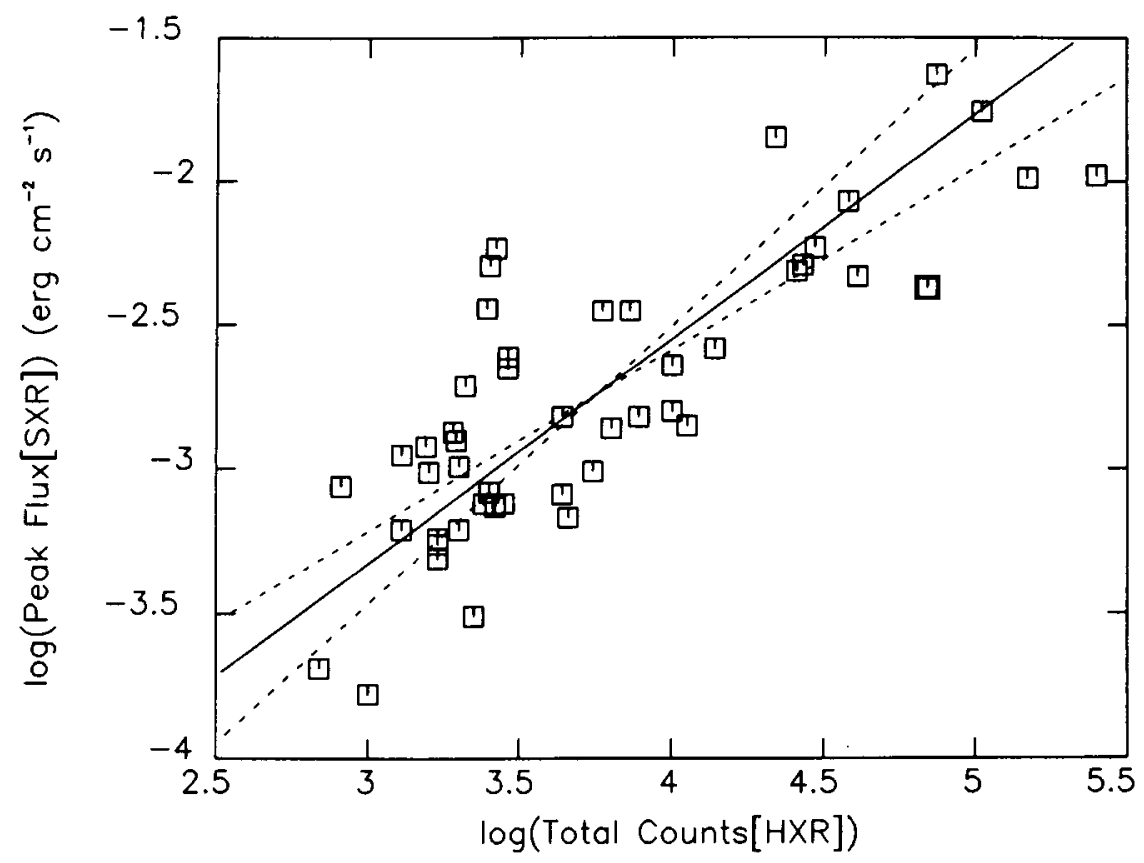

Figure 3. Plot of the log of the peak soft X-ray flux versus the log of the total hard X-ray counts. The best fit lines are as described in the caption to Figure 1.

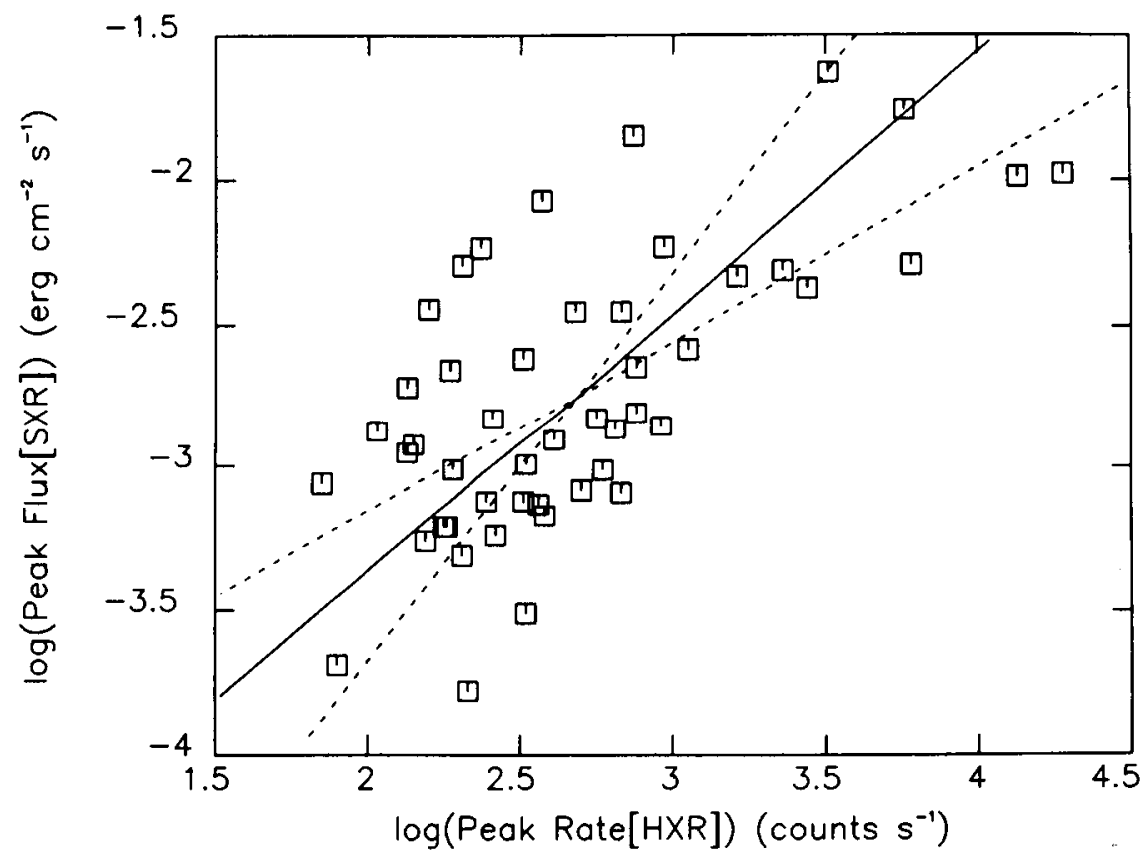

Figure 4. Plot of the log of the peak soft X-ray flux versus the log of the peak hard X-ray flux. The best fit lines are as described in the caption to Figure 1. 
regression line is determined by an iterative procedure which first employs weights for all the $\mathrm{y}$ parameters and for all the $\mathrm{x}$ parameters determined from the best-fit, one-dimensional regression lines with the alternate parameters as independent variables. On the second and any subsequent iterations, relative weights are determined from the one-dimensional rms deviations of the points from the best-fit, two-dimensional regression line. In the present study, this procedure converged after just two iterations for each pair of tested parameters. The values of $a$ and $b$ determined in this way represent the linear relationship between the two tested parameters which yields the minimum rms deviation of the sum of the squares of the perpendicular distances of the points from that line.

For the correlation, $n \cdot U_{\text {therm }}[S X R]$ with $U_{\text {beam }}[H X R]$, shown in Figure 1, the energy associated with the hard X-ray emission is determined under the assumptions that its spectrum can be represented by a single power law during each observing interval throughout the burst and that this radiation results from electrons incident on a thick target of ambient solar material. For the correlation, $n \cdot U_{t h e r m}[S X R]$ versus $U_{\text {therm }}[H X R]$, shown in Figure 2, the energy associated with the hard $\mathrm{X}$-ray emission is determined under the assumption that this radiation is due to thermal bremsstrahlung from a source with a volume that is determined from the burst rise time and ion sound speed as discussed previously. (See Equations 7, 8, and 9.) The correlations shown in Figures 3 and 4, Peak Flux[SXR] versus Total Counts[HXR] and Peak Flux[SXR] versus Peak Rate[HXR], are purely empirical and do not involve any interpretative model. 


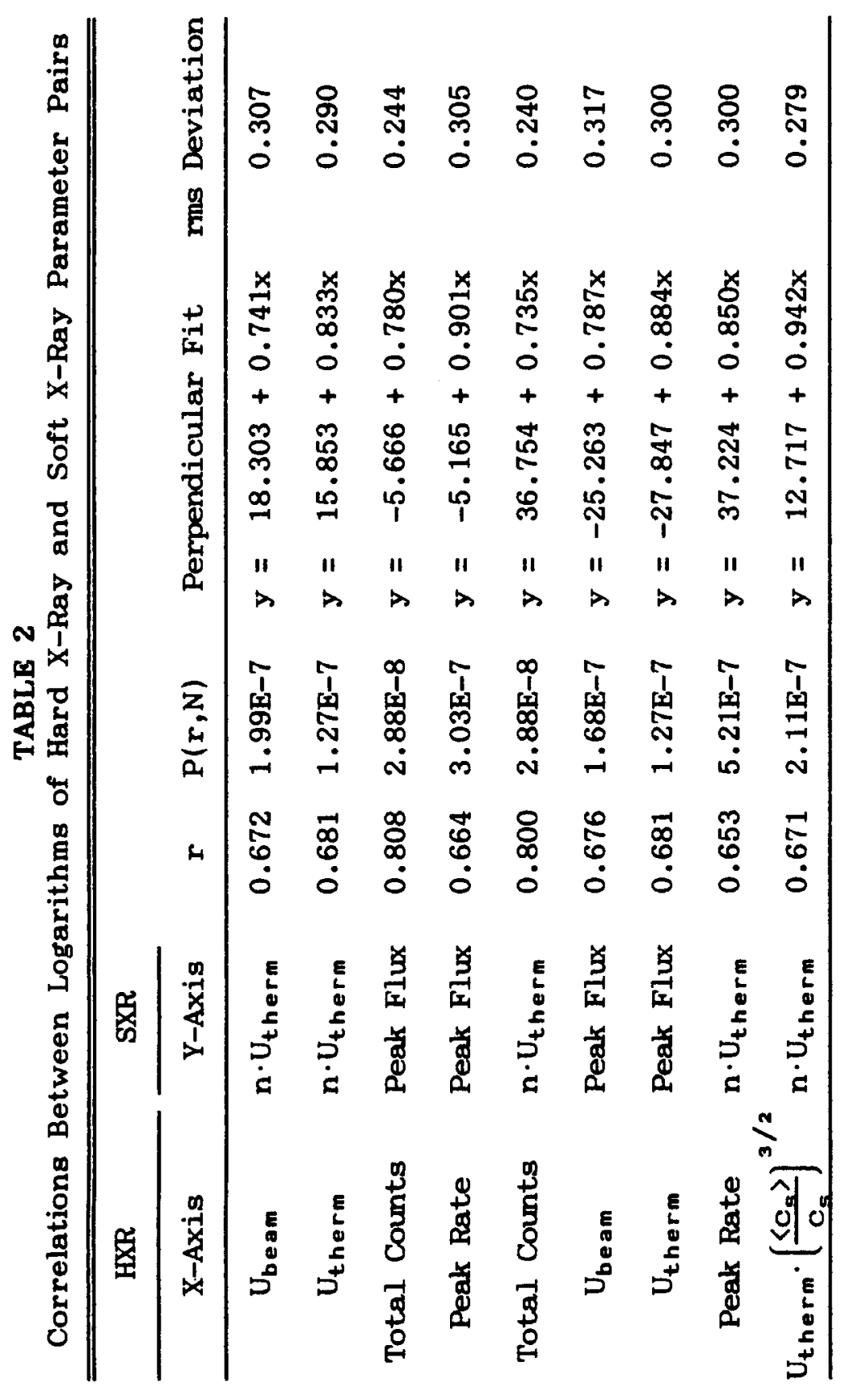


The results presented in Table 2 demonstrate that each of these four parameter pairs is correlated to a highly significant degree. This confirms, with a much larger data set and, hence, with much greater statistical significance, the energetics relationship originally reported by CKT. As a discriminator between flare models, however, this test contributes nothing to distinguishing between thermal and nonthermal models of impulsive flare emissions. Of these four, in fact, the only correlation that is significantly different from the others is between the model-independent parameters Peak Flux[SXR] and Total Counts[HXR]; and this correlation is distinctly better than those based on models.

Results of the correlation analyses for five additional parameter pairs also are presented in Table 2. Four of these are permutations of the model-dependent parameters with the model-independent parameters. The results are seen to be independent of whether the Peak Flux or $n \cdot U_{\text {therm }}[S X R]$ are chosen to represent the soft X-ray energetics; and, again, Total Counts[HXR] yields a significantly better correlation than either of the two model-dependent representations of the hard X-ray energetics. The final correlation analysis, between $n \cdot U_{t h e r m}[S X R]$ and $\mathrm{U}_{\text {therm }} \cdot\left[\left\langle\mathrm{c}_{\mathrm{s}}\right\rangle / \mathrm{c}_{\mathrm{s}}\right]^{3 / 2}[\mathrm{HXR}]$, was carried out in order to determine if the use of the actual ion sound speed determined for each event, rather than an average value characteristic of the whole set, resulted in a better correlation. A comparison of the results for $U_{t h e r m} \cdot\left[\left\langle c_{s}\right\rangle / c_{s}\right]^{3 / 2}[H X R]$ in which an average value of the ion sound speed is substituted for the individual values with the results for $U_{\text {therm }}[H X R]$ indicates that there is no significant difference. 
It should be noted that the correlation between Peak Flux[SXR] and Peak Rate[HXR] reported here is significantly better than that reported by CKT. This may be explained by the much larger dynamic range covered by the events in this study (2.4 decades as compared to 1.2 decades in the Peak Rate[HXR]) and by the much smaller sample size available for the earlier work ( 8 events as compared to 48 events in the present study). The value of the correlation coefficient reported here for the Peak Flux[SXR] versus Peak Rate[HXR] is of greater statistical significance both because of the greater number of events and because of smaller scatter in the distribution, by a factor of $\sim 1.3$. The two data sets are otherwise consistent with originating from a common population.

Taken all together, the results of these correlation analyses suggest that their inability to distinguish between models is at least in part a result of the limited nature of the observations. Calculations of $U_{\text {therm }}[H X R]$, for example were hindered by the lack of microwave observations that necessitated the use of the hard $X$-ray burst rise time to estimate source volume as derived in Equations (7) through (10). An estimate of the uncertainty this introduces in the values of $U_{t h e r m}[H X R]$ can be obtained from the scatter in the correlation between measured and theoretical rise times reported by BCWM. Two different uncertainties in the details of the source geometry contributes to that scatter. If all of it were due to variations in $\eta$, with corresponding variations in the constant of proportionality between $\mathrm{L}^{3}$ and source volume, then the use of burst rise time to estimate source size would introduce an $r$ s scatter of a factor of 2 in the values of $U_{t h e r m}[H X R]$, 
or 0.3 in their logarithms. As can be seen in Table 2, this alone is sufficient to account for all the scatter found between $U_{t h e r m}[H X R]$ and other energetics parameters. More realistically, variations in the projected areas of the individual flaring loops investigated by BCWM also contribute to the scatter in their results. Thus, the result that the correlation between $n \cdot U_{t h e r m}[S X R]$ and $U_{t h e r m}[H X R]$ is weaker and yields a larger rms deviation than the correlation between Peak Flux[SXR] and Total Counts[HXR] may be due to observational uncertainties in the parametric technique employed. While the uncertainties in $U_{\text {beam }}[\mathrm{HXR}]$ are difficult to estimate quantitatively, they also may be as large as a factor of 2 (or 0.3 in the logarithm). 


\section{ION CONDUCTION FRONT MODEL}

\section{A. DESCRIPTION OF THE MODEL}

Brown, Melrose and Spicer (1979, hereafter BMS) were the first to present a thermal model of hard X-ray production in which a collisionless conduction front was invoked in order to avoid the problem of unacceptably rapid cooling. They explained that the classical heat conduction rate assumed by Kahler $(1971,1975)$ was unrealistically rapid under the nearly collision-free conditions in such a hot plasma volume. They showed that escaping electrons, which constitute the heat flow, would be retarded by interactions with ion-acoustic or ion cyclotron waves excited at the boundary. This would limit the conduction such that the boundary of the hot volume, or conduction front, would advance at a velocity of $\sim c_{s}=\left(k T_{e} / m_{p}\right)^{1 / 2}$. The classical conduction rate implies a front velocity of $\sim_{v_{e}}=\left(k T_{e} / m_{e}\right)^{1 / 2}$, so the heat flux limitation suggested by BMS reduced the conduction rate by a factor of $\left(\mathrm{m}_{\mathrm{p}} / \mathrm{m}_{\mathrm{e}}\right)^{1 / 2} \approx 43$. BMS showed that if such a hot parcel of plasma were produced by impulsive heating at the apex of a magnetic coronal arch, then the lifetime of the source would be $\sim L / c_{s}$, where $L$ is the half-length of the arch. $L / c_{s} \equiv \tau_{s}$ is the time required for the source boundaries to reach the arch footpoints, where contact with high-density, much cooler plasma would cool the source at a correspondingly higher rate. In the BMS model, the rise time of a burst is not considered, but is presumed to be of order $\tau_{A}=1 / v_{A}$, where $\ell$ is the characteristic size of heated kernels and $v_{A}$ is the Alfven velocity; this is based on the assumption that magnetic reconnection is the mechanism of heating. In the thermal model of BMS, the timescale, $\tau_{s}=\mathrm{L} / \mathrm{C}_{s}$, is predicted to be the fall time of the burst. 
Smith and Lilliequist (1979) tested the BMS physical model with a fluid simulation, but found that continuous input of heat was necessary to sustain a hard X-ray source. In their version of the model, and in later papers by Smith and Harmony (1982) and Smith and collaborators (see Smith 1986 and references therein) dealing with improved simulations, values of the heating time $\tau_{H}$ greater than $L / c_{s}$ were used. This adaptation of the model bears an important distinction from the BMS version: the burst rise time is given by $\tau_{5}=\mathrm{L} / \mathrm{c}_{5}$, not the fall time, because continuous heating increases the hard $\mathrm{X}$-ray flux until it is overcome by enhanced cooling through contact between the source and the chromosphere.

\section{B. IMPLICATIONS OF THE MODEL FOR HARD X RAYS}

In Batchelor (1984) and Batchelor et al. (1985, hereafter BCWM) a new adaptation of the conduction front model was presented which made several important revisions to the existing model. BCWM argued, in contrast to previous works (e.g. Smith and Brown 1980), that the Maxwellian tail of the electron distribution could be populated up to the energies necessary for microwave emission, that a sufficient number of electrons would have pitch angles great enough to be efficient producers of microwaves, and that they would be confined by the thermoelectric field or magnetic mirroring. Also in BCWM, the lead of Smith and collaborators was followed, and it was the rise time, $t_{r}$, of the impulsive hard $\mathrm{X}$-ray burst that was equated to $\mathrm{L} / \mathrm{c}_{\mathrm{s}}$ for a thermal model with conduction front confinement and continuous heating. For 23 flares, BCWM computed $L$ from the microwave and hard X-ray data and 
$c_{s}$ from the hard $\mathrm{X}$-ray spectra. The plot of $\tau_{s}=\mathrm{L} / \mathrm{c}_{s}$ versus burst rise time, $t_{r}$, strongly suggested that $t_{r} \approx \tau_{s}$, in agreement with the revised model.

These revisions to the model have two particularly significant implications. First, the confinement of the tail electrons of this thermal distribution behind the conduction front implies that a common population of electrons is responsible for both the hard X-ray and the microwave emissions. This is a very strong statement because it requires that the electrons producing these two emissions come not just from the same loop but from the same region of the loop so that fundamental physical parameters such as magnetic field, electron density, electron volume, and electron temperature simultaneously characterize both the hard $\mathrm{X}$-ray and the microwave emitting regions. Secondly, the implied equality between the measured rise time, $t_{r}$, and the theoretical quantity, $\mathrm{L} / \mathrm{c}_{\mathrm{s}}$, enables a direct observational test of the model to be made.

In order to extract the loop length from the hard $X$-ray and microwave data, BCWM followed the procedure of Crannell et al. (1978). Here it is assumed that the microwave flux, $S(f)$, peaks at some frequency, $f_{\max }$, and that at frequencies $f<f_{\max }$ the microwave emission is optically thick and the spectrum is given by the Rayleigh-Jeans law:

$$
S(f)=0.16 f^{2} A T,
$$

where $S$ is the microwave flux at the Earth in solar flux units, $f$ is the 
frequency in $\mathrm{GHz}, \mathrm{A}$ is the observed source area in $10^{18} \mathrm{~cm}^{2}$, and $\mathrm{T}$ is the temperature in $\mathrm{keV}$. One can then calculate $A$ with values for $f$ and $S(f)$ from the optically thick portion of the microwave spectrum and a corresponding value for $T . \quad T$ is obtained by fitting a thermal bremsstrahlung function to the hard $\mathrm{X}$-ray data as described previously.

The ion sound speed, $c_{s}$, is easily obtained from the temperature:

$$
c_{s}=3.1 \times 10^{-2} \mathrm{~T}^{1 / 2}
$$

where $c_{s}$ is in units of $10^{9} \mathrm{~cm} \mathrm{~s}^{-1}$ and $\mathrm{T}$ is given in keV.

The area, A, determined from Equation 20 may not be identically equal to the actual area of the loop. A given arch, if viewed from the side, has a projected area of $2 \mathrm{Lw}$, where $\mathrm{L}$ is the half-length of the loop and $w$ the width. As BCWM pointed out, however, the observed projected area depends on such unknown quantities as the arch dimensions, arch orientation and anisotropic microwave emission, thus producing the inequality, $A \leqslant 2 L w \leqslant 2 \pi A$, which is expected to hold for every burst. The unknown relationship between the arch dimensions can be parameterized as $\eta=2 \mathrm{~L} / \mathrm{w}$, where $\eta$ is the ratio of the total length of the loop to its average width. Substituting for $w$ in the inequality and taking the square root yields Equation 8 of BCWM:

$$
(\eta \mathrm{A})^{1 / 2} / 2 \leq \mathrm{L} \leq(\pi \eta \mathrm{A} / 2)^{1 / 2},
$$

where 


$$
L=L(t)=\int^{t} c_{s}\left(t^{\prime}\right) d t^{\prime}
$$

One should be reminded at this point that BCWM determined the area only at the peaks of their 23 events. They defined the area of a loop at the peak of a burst, as determined from the model, to be $A_{0}$, and the corresponding half-length and rise time to be $\mathrm{L}_{0}=\mathrm{A}_{0}^{1 / 2}$, and $\tau_{0}=\mathrm{L}_{0} / \mathrm{c}_{5}$, respectively. In this analysis, the length $L(t)$ is the distance along the loop that has been swept out by the conduction front moving at a velocity equal to the ion sound speed, $c_{s}$, as indicated in Equation 23. If we assume that the width, $w$, is nearly constant throughout the loop then the area of the loop, $2 \mathrm{Lw}$, the half-length, $\mathrm{L}$, and the geometric parameter, $\eta$, all grow as a function of time in the same way. And because $\eta$ is the only free parameter in the model it is useful to rewrite Equation 22 as follows:

$$
4 \mathrm{~L}^{2} / \mathrm{A} \geq \eta \geq(2 / \pi) \mathrm{L}^{2} / \mathrm{A}
$$

Both of these limits correspond to views of loops observed on the limb, with the smallest value occurring when the line of sight is in the plane of the loop curvature and the largest value when the line of sight is perpendicular to that plane. A loop on the disk would have an intermediate value.

\section{OBSERVATIONAL DATA}

As described in Section II.B., an initial set of 118 simple impulsive spike bursts was compiled from a thorough search of SMM/HXRBS 
observations between February 1980 and December 1982. A total of six of these passed all of our requirements on the quality of the X-ray data and also had adequate microwave coverage to support a detailed investigation of the ion conduction front model. The microwave frequencies available for these six events are shown in Table 3. Note that two of the six (HXRBS events 674 and 1456) could not be included in the energetics study described in Section II because adequate soft $\mathrm{X}$-ray coverage was not available for them.

In Figure 5, time histories of the hard X-ray and microwave observations are displayed for three of the events used in the model test. The microwave data available for each event varied. The frequencies at which microwave observations are generally available from the Bern Radio Telescopes include 8.4, 10.4, 11.8, 19.6 and $35.0 \mathrm{GHz}$; however, most of these events were not observed at all five of these frequencies. The time resolution of the hard X-ray and microwave data displayed in Figure 5 is $1.024 \mathrm{~s}$, and the two data sets register to better than $64 \mathrm{~ms}$.

\section{RESULTS OF THE MODEL TEST}

In Figure 6, we show the data presented in Figure 7 of BCWM plus the corresponding data for the events employed in our test of the model. The squares are their 20 events and the circles are our six events. The two solid pairs of data points connected by lines are events that are common to both of these investigations. The pair within the dashed lines is the flare of 1980 March 29, peaking at about 0918 UT (Event 87 in Tables 3 and 4 and Event Number 1 in BCWM), and the 


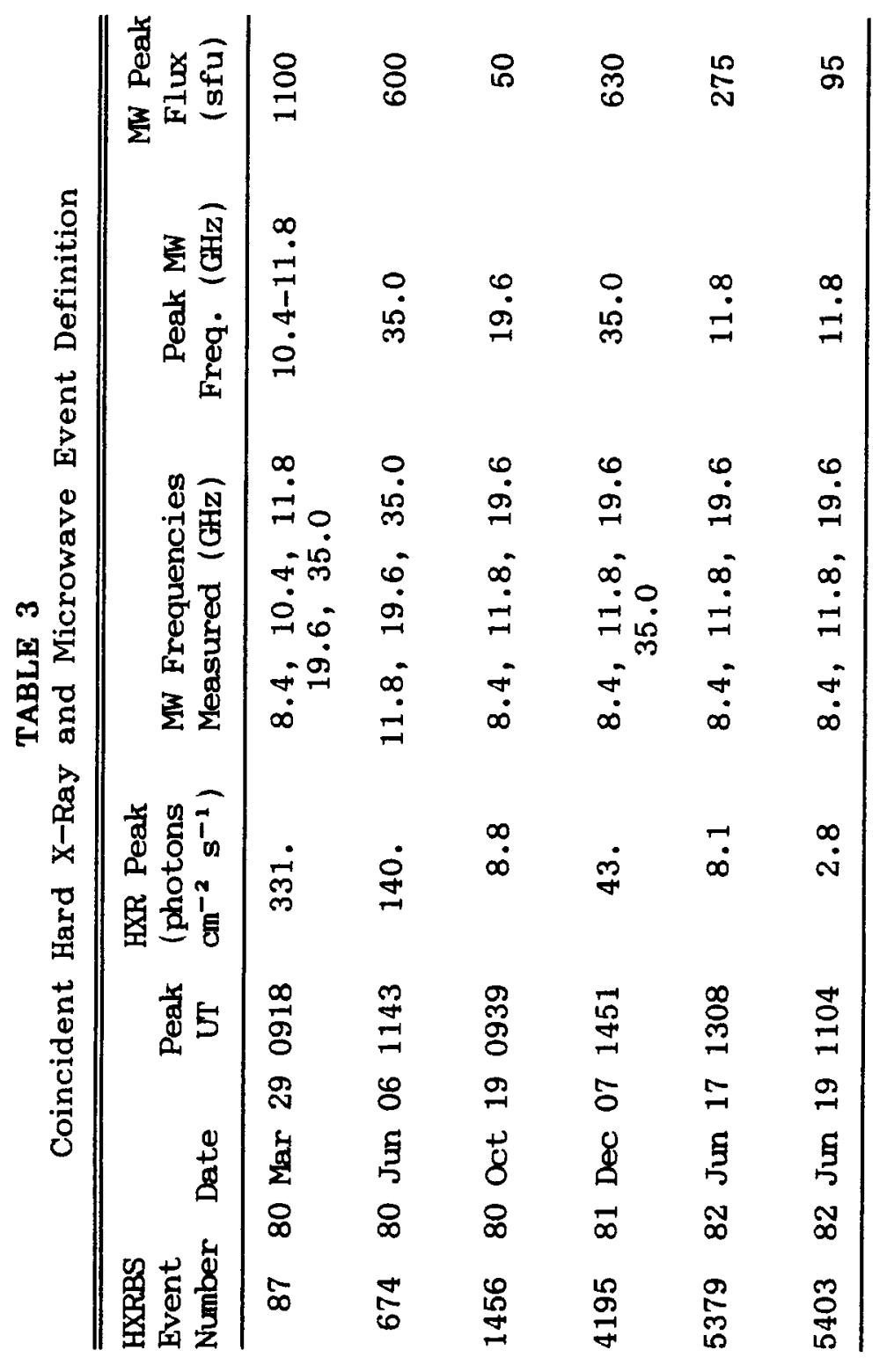


ORIGINAL PAGE IS

OA POOR QUALITY
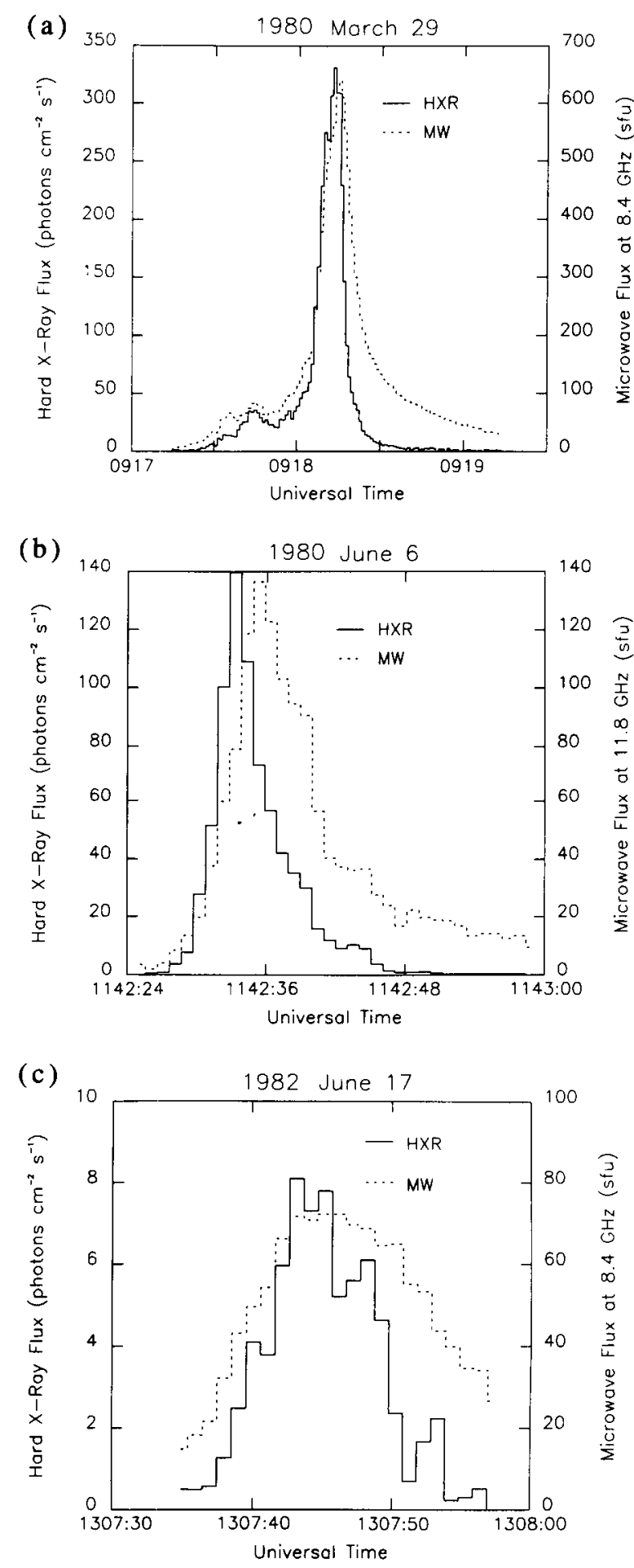

Figure 5. Time histories of the hard X-ray flux from a thermal analysis of the HXRBS observations (solid line) and microwave flux at the lowest available frequency from the Bern Radio Observatory (dashed line) for (a) 1980 March 29, (b) 1980 June 6, and (c) 1982 June 17 events. The time resolution in this representation is $1 \mathrm{~s}$. 


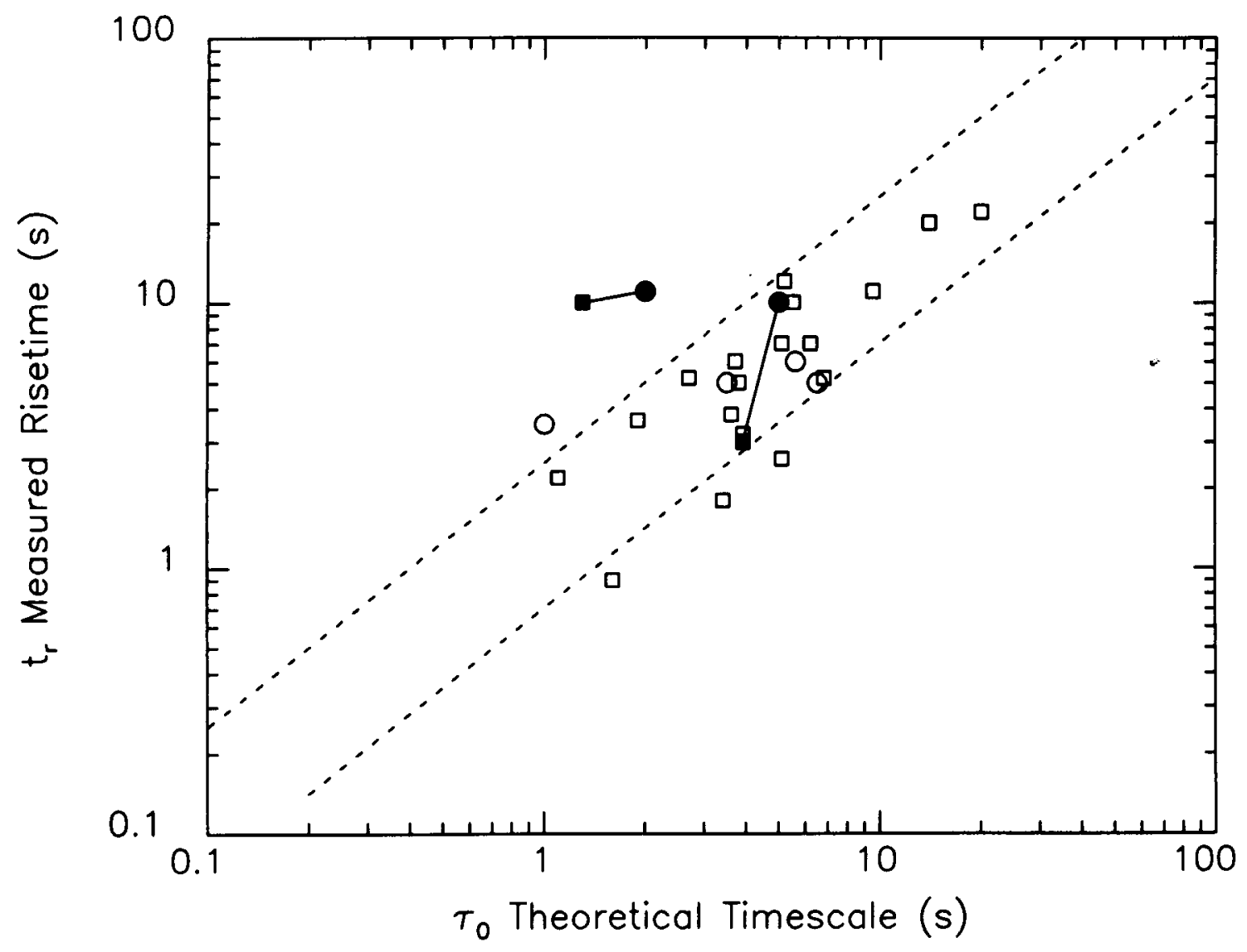

Figure 6. Plot of the log of the measured rise time of the hard X-ray burst versus the log of the theoretical rise time for 20 events from BCWM (squares) and our six events (circles). The dashed lines (also from $\mathrm{BCWM}$ ) are boundaries of the expected positions on the plot, if the sources are arches from 2 to 4 times as long as they are wide. The two pairs of connected points designate events that the two data sets have in common. 
other pair is the flare of 1981 December 7, peaking at about 1452 UT (Event 4195 in Tables 3 and 4 and Event Number 23 in BCWM). Though there is some disagreement between BCWM and the present work as to the exact placement of these two events on the plot, the differences are negligible for the purposes of this analysis. The significance of Figure 6 is that the six events selected for this study follow the same trend found by BCWM and are consistent with membership in the same population.

In Figure 7, the area, $A$, inferred from the Rayleigh-Jeans law (Equation 21) is plotted against the integral of the ion sound speed, $c_{s}$, over time. For each plot in Figure 7 time advances from left to right in 1-s intervals. The zero for the integral is arbitrary. The line through the data shows the fit to those points that we took to represent the time interval from the start to the peak of each flare in hard $\mathrm{X}$ rays. $\Delta \mathrm{A}$ is the change in area over this time and $\Delta \mathrm{L}$ is the corresponding change in the loop length. In order to agree with the BCWM model, when the quantity $(\Delta \mathrm{L})^{2} / \Delta \mathrm{A}$ is inserted into Equation 25 , one must obtain a reasonable value of $\eta$, the geometric factor. It is generally accepted that $\eta$ is of order 10 (cf. Colgate 1978, Van Hoven 1981, and BCWM). As shown in Table 4, the events that have been studied here are consistent with such values. In only one of the six events (4195) does $\eta$ exceeds the typical range. This may be attributable to partial occultation of that source, which was the only one that occurred on the limb.

Previously published results for this same set of events (Starr et al. 1986) reported disagreement between the observational test and the 


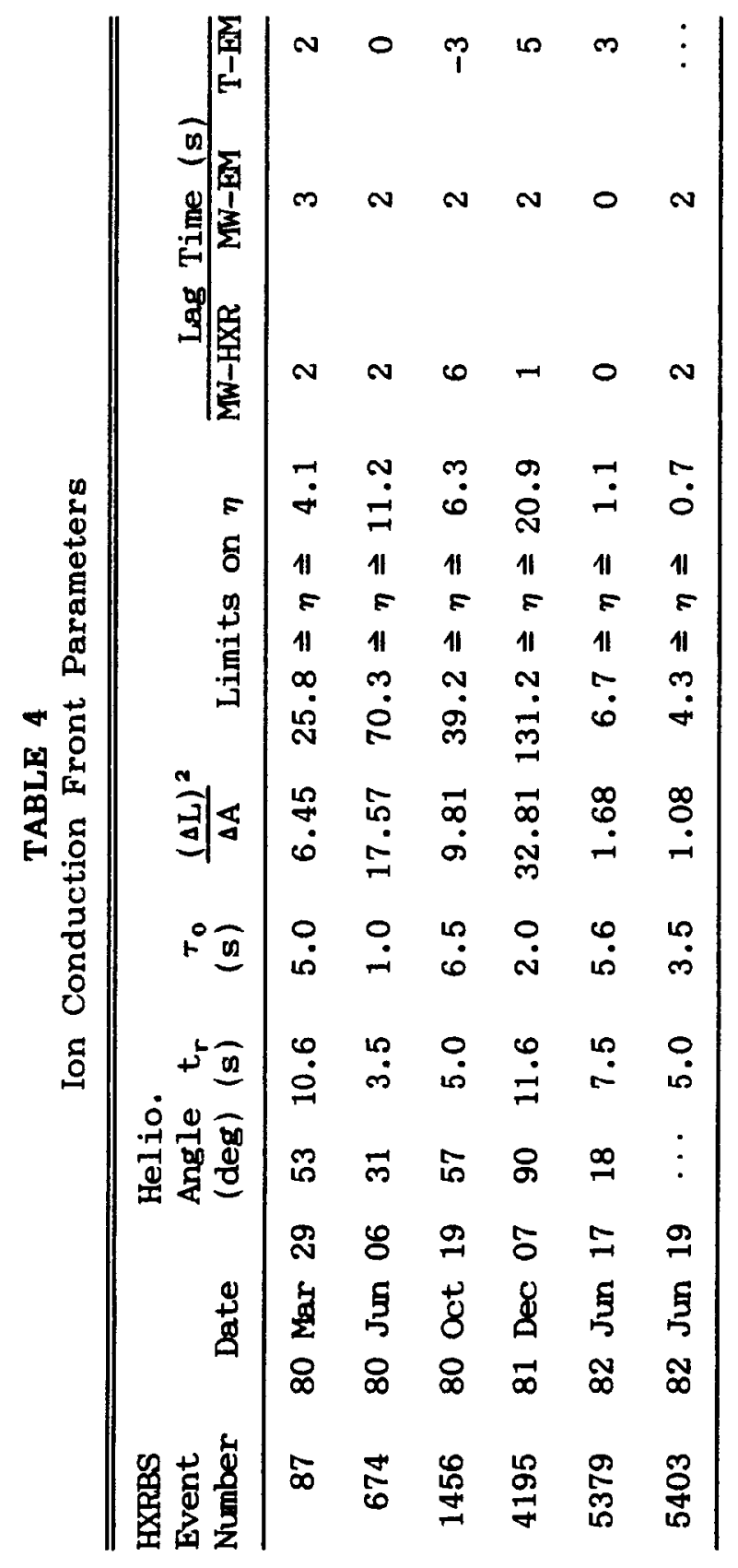



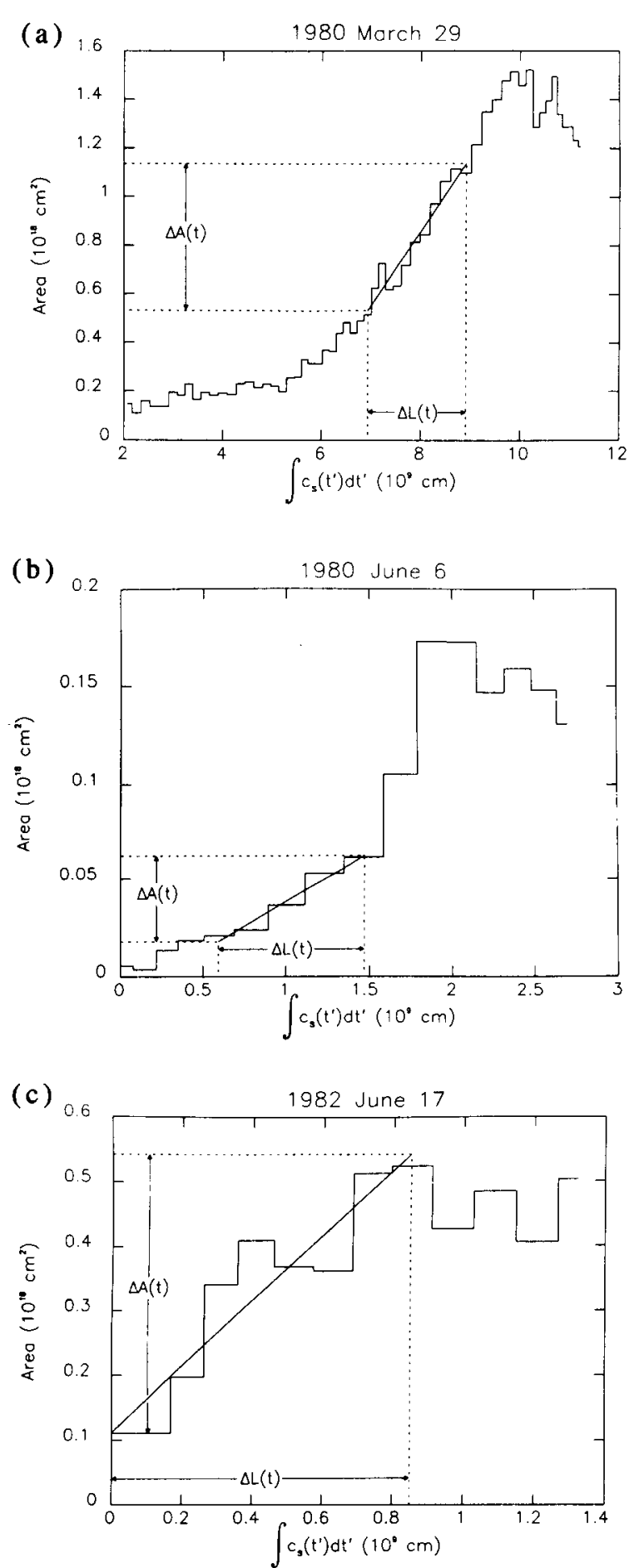

Figure 7. Loop area A, versus the integral over time of the ion sound speed, $\mathrm{c}_{\mathrm{s}}$, for (a) $1980 \mathrm{March} 29$, (b) 1980 June 6, and (c) 1982 June 17 events. Time advances from left to right in 1-s intervals. The zero for the integral is arbitrary. The line through the data shows the fit to those points which we took to represent the time interval from the start to the peak of each $\mathrm{X}$-ray burst. $\Delta \mathrm{A}$ is the change in area over this time and $\mathrm{AL}$ is the corresponding change in the loop length. 
model. In that analysis, the square root of the area was assumed to vary in time in the same way as the length, thus implying that the width also varied in time and that $\eta$ was constant. Such a picture of flare geometry and loop growth is, we believe, less realistic than that chosen for the present analysis, where $\mathrm{dA} / \mathrm{dt} \approx \mathrm{dL} / \mathrm{dt}=\mathrm{c}_{\mathrm{s}}$.

\section{E. POST PEAK SPECTRAL DYNAMICS AND IMPLICATIONS FOR THE CONDUCTION FRONT MODEL}

In Figure 5, the $\mathrm{X}$-ray and microwave time histories clearly display a time lag between the peak of the $X$ rays and the peak of the microwave emission for two of the events and no time lag for the third. The maximum time lag for the six events listed in Table 4 is about $6 \mathrm{~s}$ and the average is about 2 s. A similar relationship between hard X-ray and microwave peak time has been reported by Crannell et al. (1978), who found an average time lag of $\sim 2 \mathrm{~s}$ in 22 simple impulsive spike bursts. On the other hand, Cornell et al. (1984) found an average time lag of $\sim 0.2 \mathrm{~s}$ for 11 sharp features contained in five separate flares. In that work, an effort was made to select flares with sub-second time structure in hard $X$ rays and to see if the same structure was also present in microwaves. In the present work and in Crannell et al., simple spike bursts were chosen, and flares with multiple impulsive spikes that might have exhibited the sub-second time structure were not included. In addition, Cornell et al. measured microwave emission at only one frequency $(10.6 \mathrm{GHz})$, and so whether or not these emissions are in the optically thick or thin part of the microwave spectrum cannot be determined. For all of the events in Crannell et al. and for the six events in this work with good microwave coverage, optically thick 
microwave data are available, and it is the time histories of these that are compared to the hard X-ray emission.

The difference between the peak time of a hard X-ray burst and the peak time of its associated microwave emission is not addressed directly by the BCWM model. The physical considerations on which the model is based, however, provide a context in which to interpret the observations. When a conduction front reaches the footpoints of a loop, it is disrupted by the dense, cool plasma of the chromosphere. Subsequently, only one of the two previously operative trapping mechanisms, namely, magnetic mirroring, continues to confine the energetic electrons within the loop. As a result, both the volume of a source and the total number of energetic electrons it contains achieve their maximum values simultaneously, at the time the conduction front impacts the footpoints. The temperature, on the other hand, continues to be influenced by competing processes: first, by any continued heating of the loop and, second, by cooling due to upflowing ambient material from the footpoints and/or by loss of energetic electrons.

The hard X-ray emission is optically thin and, for small changes in the temperature, influenced predominately by the emission measure. This emission, therefore, should peak at the time the conduction front reaches the footpoints of the flaring loop. The microwave emission, however, is optically thick and relatively insensitive to the emission measure. It, therefore, should respond predominately to the temperature of the source region. The set of flares analyzed in detail in this work reflects the diversity of possible outcomes of these competing processes. 
For these bursts, as well as for the simple impulsive spike bursts investigated by Crannell et al. (1978), the hard X-ray spectrum appears to be thermal during the rise and at the peak of each burst. The best-fit temperature exhibits a soft-hard-soft behavior, increasing during the rise and decreasing during the fall of each burst. During the falling portion, however, the hard X-rays also exhibit a spectral hardening leading to the development of a power-law tail that, in the highest energy HXRBS channels, lies above the best-fit thermal spectrum. Just such a spectral hardening is expected for a population of energetic electrons trapped in a thick target and losing energy by interactions with a cooler distribution of particles (Takakura and Kai 1966). The ion conduction front model as refined by BCWM is consistent with this observation. At burst maximum, the plasma wave-electron interactions, which operate as the thermalizing process within the conduction front, stop because the conduction front no longer exists. Thereafter, the electron distribution is in essence nonthermal, because there is no longer an equilibration process between the high-energy tail of the distribution and the slow electrons in the bulk of the distribution. Thus, the somewhat anomalous spectral evolution is consistent with the speculation that the initial population of energetic electrons dissipates its energy in cool chromospheric material after disruption of the conduction front.

For all but one of the events, the temperature determined from the hard X-ray observations peaks at the same time or somewhat later than the corresponding emission measure. (See Figure 8.) For all of the 
events, the optically thick microwave emission also peaks at the same time or later than the associated hard X-ray emission and falls more slowly. The common source hypothesis, for hard X-ray and microwave emission, can be tested in detail from event-by-event comparisons between the lag time of the microwave emission and the lag time of the temperature, each with respect to the peak emission measure. As can be seen in Table 4, they are not consistent or correlated with each other, despite the tendency of both to be positive. These results suggest a scenario in which, after disruption of the conduction front, hard X-rays are produced primarily at the footpoints of the flaring loop, while the microwave emission is produced primarily at the loop top. Differences between the detailed time histories of the microwave emission and the temperature determined from the hard X-ray observations could then be explained by variations in the post-maximum heating and cooling rates in the respective source regions.

This analysis of spectral dynamics also provided a ready means for testing one prediction of Matzler et al. (1978) for the adiabatic compression model, namely, that the reversible relationship $E M \propto T^{3 / 2}$ holds throughout single impulsive spike bursts. Elcan (1978) and Wiehl et al. (1985) found that no such simple relationship characterized the events that they studied, but in both of those studies the flare selection criteria were less restrictive than in the present work. We have examined the temperature-emission measure variations for the six events listed in Table 3 in order to learn whether the diverse relationships found previously could be accounted for by assuming that those studies actually included complex or composite bursts. However, 
we, too, find that there is no simple relationship that fits all of our well-selected events, as can be seen in Figure 8 . Thus, even in a set of single impulsive spike bursts, the prediction that the temperature and emission measure should exhibit characteristics of a reversible process is violated. 


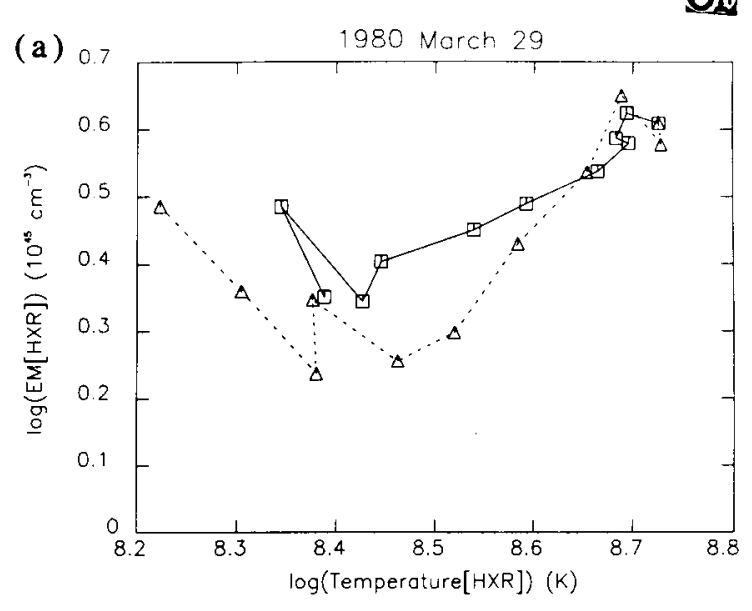

OE ROOR QUALITY
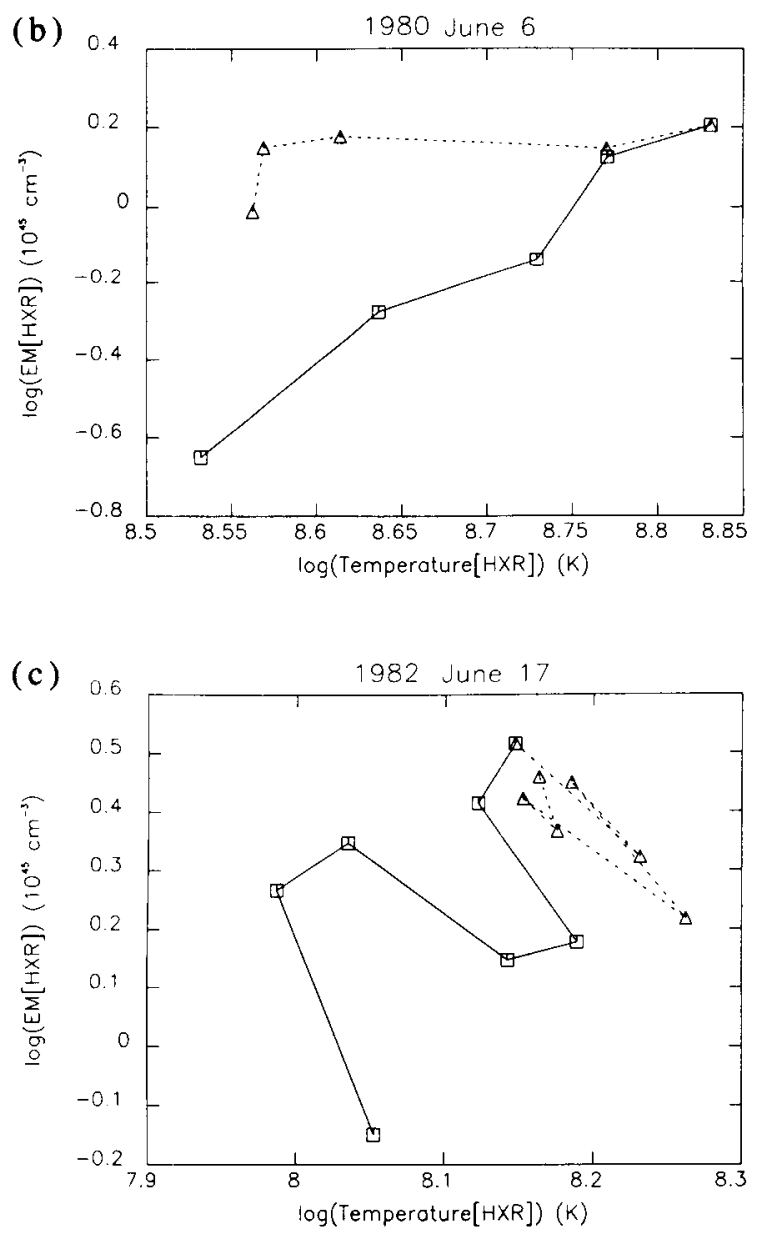

Figure 8. Plot of the log of the emission measure versus the log of the temperature from thermal fits to HXRBS data for (a) 1980 March 29, (b) 1980 June 6, and (c) 1982 June 17 events. The squares (connected by solid lines) are on the rise of the flare and the triangles (connected by dashed lines) are on the fall. 


\section{CONCLUSIONS}

The thermal model and the thick-target, beam model of the impulsive emissions both yield strong correlations between the energetics of the impulsive and the gradual emissions. Such a correlation is necessary but not sufficient evidence for a causal relationship. There is, however, further evidence to support the hypothesis that the relationship is causal and, in particular, that the source of the impulsive emissions supplies the energy that powers the gradual emissions. One is the result, first reported by $\mathrm{CKT}$ and supported by the present work, that the energies in the sources of the associated impulsive and gradual emissions are essentially equal for densities of the soft $X$-ray emission source of approximately $10^{11} \mathrm{~cm}^{-3}$. The densities implied by the two models differ only by a factor of 1.5 , and the central values and the range of values implied by both are consistent with the allowable range of values reported by Dere et al. (1979) and Feldman, Doschek, and Kreplin (1980) based on independent considerations. For the beam model with a power-law spectral analysis, it might be argued that the value found for the implied density is fortuitous because the calculated energy can be changed by orders of magnitude through selection of the low energy cutoff. The finding that the thermal model yields the same result with a parametric analysis of all the impulsive hard X-ray emission, however, refutes this argument.

Another supporting piece of evidence is the observation by Neupert (1968) that, for an individual burst, the soft X-ray emission varies with time as the integral of the hard $\mathrm{X}$-ray burst profile. This temporal relationship has been verified in detail for two impulsive events by 
Tanaka et al. (1983). While it is possible that these suggestive results are merely coincidental and the correlation is merely a manifestation of the as yet unexplained big-flare syndrome (Kahler 1982), the weight of the evidence argues strongly in favor of pursuing more definitive tests of the hypothesis. Such a test can be accomplished with measurements of density-sensitive line emission in coincidence with hard X-ray and microwave observations of the impulsive components.

As discussed in Section II.C., scatter in the correlations between hard and soft X-ray energetics parameters used for our study is an unavoidable consequence of the scatter introduced by inherent uncertainties in the burst geometry. In future studies of these relationships, spatially resolved hard X-ray observations and density measurements for the associated soft X-ray source would certainly provide much more powerful solar-flare diagnostics.

The correlational analysis employed in the present work does not distinguish between thermal and nonthermal models of impulsive emissions, or even between models and a model-independent interpretation. While an analysis of flare energetics is a useful tool, we have found that simply looking at the peak values of the hard $X$-ray parameters fails to shed any new light on the flare problem. An understanding of the dynamical evolution of impulsive solar flares is essential, and any model that attempts to explain the hard $\mathrm{X}$-ray emission must address this issue.

The simple ion conduction front model successfully describes the 
growth of a source in a flaring loop during the rise of a hard X-ray burst. For five out of the six events studied here, the growth of the hard $\mathrm{X}$-ray/microwave emitting region is well correlated with a scale length that increases at a rate determined by the ion sound speed. The fundamental assumption of a common source for the hard X-ray and microwave emission that this and other thermal models share is supported by this analysis. In addition, it appears that the the BCWM model may provide a context in which to understand the time lag between the peak of the hard X-ray flux and the associated microwave emission. 


\section{REFERENCES}

Anderson, K. A., and Winkler, J. R. 1962, J. Geophys. Res., 67, 4103.

Batchelor, D. A. 1984, Ph.D. Dissertation, University of North Carolina,

Chapel Hill, and NASA Technical Memorandum 86102.

Batchelor, D. A., Crannell, C. J., Wiehl, H. J., and Magun, A. 1985, Ap. J., $295,258$.

Bevington, P. R. 1969, Data Reduction and Error Analysis for the

Physical Sciences, (New York: McGraw-Hill).

Brown, J. C. 1971, Solar Phys., 18, 489.

Brown, J. C. 1975, Solar Gamma-, $X-$, and EUV Radiation, ed. S. R. Kane

(Dordrecht: Reidel), p. 245.

Brown, J. C., Melrose, D. B., and Spicer, D. S. 1979, Ap. J., 228, 592.

Brown, J. C., Craig, I. J. D., and Karpen, J. T. 1980, Solar Phys., 67, 143.

Brown, J. C., Carlaw, V. A., Cromwell, D., and Kane, S. R. 1983, Solar Phys., 88, 281.

Chubb, T. A., Kreplin, R. W., and Friedman, H. 1966, J. Geophys. Res., 71, 3611.

Colgate, S. 1978, Ap. J., 221, 1068.

Cornell, M. E., Hurford, G. J., Kiplinger, A. L., and Dennis, B. R. 1984, Ap. J., 279, 875.

Crannell, C. J., Frost, K. J., Matzler, C., Ohki, K., and Saba, J. L. 1978, Ap. J., 223, 620.

Crannell, C. J., Karpen, J. T., and Thomas, R. J. 1982, Ap. J. 253, 975. Dennis, B. R., Orwig, L. E., Kiplinger, A. L., Gibson, B. R., Kennard, G. S., and Tolbert, A. K. 1985, NASA Technical Memorandum 86236. Dere, K. P., Mason, H. E., Widing, K. G., and Bhatia, A. K. 1979, Ap. J. 
Suppl., 40, 341.

Duijveman A., Hoyng, P., and Machado, M. E. 1982, Solar Phys., 81, 137.

Elcan, M. J. 1978, Ap. J. (Letters) 226, L99.

Feldman, U., Doschek, G. A., and Kreplin, R. W. 1980, Ap. J., 238, 365.

Gary, D. E., Hurford, G. J. 1986, Bull. Am. Astr. Soc. 18, 900.

Hoyng, P.,Brown, J. C., and van Beek, H. F. 1976, Solar Phys., 48, 197.

Kahler, S. 1971, Ap. J., 164, 365.

Kahler, S. 1975, Solar Gamma-, $X-$, and EUV Radiation, ed. S. R. Kane

(Dordrecht: Reidel), p. 211.

Kahler, S. W. 1982, J. Geophys. Res., 87, 3439.

MacKinnon, A. L., Brown, J. C., and Hayward, J. 1985, Solar Phys., 99, 231.

Matzler, C., Bai, T., Crannell, C. J., and Frost, K. J. 1978, Ap. J. 223, 1058.

Neupert, W. M. 1968, Ap. J. (Letters), 153, L59.

Peterson, L. E., and Winkler, J. R. 1959, J. Geophys. Res., 64, 697.

Smith, D. F., and Lilliequist, C. G. 1979, Ap. J., 232, 582.

Smith, D. F., and Brown J. C. 1980, Ap. J., 242, 799.

Smith, D. F., and Harmony, D. W. 1982, Ap. J., 252, 800.

Smith, D. F. 1986, Ap. J. 302, 836.

Starr, R., Crannell, C. J., Thomas, R. J., Batchelor, D. A., and Magun, A. 1986, Bull. Am. Astr. Soc., 18, 965.

Takakura, T., and Kai, K. 1966, Pub. Astr. Soc. Japan, 18, 57.

Tanaka, K., Nitta, N., Akita, K., and Watanabe, T. Solar Phys., 86, 91.

Thomas, R. J., Starr, R., and Crannell, C. J. 1985, Solar Phys. 95, 323.

Wiehl, H. J., Schochlin, W. A., and Magun, A. 1980, Astron. Astrophys. 92, 260. 
Wiehl, H. J., Batchelor, D. A., Crannell, C. J., Dennis, B. R., Price, P. N., and Magun, A. 1985, Solar Phys. 96, 339.

Van Horn, G. 1981, Solar Flare Magnetohydrodynamics, ed. E. R. Priest, (New York: Gordon and Break), Chapter 4.

Wu, S. T., de Jager, C.,Dennis, B. R., Hudson, H. S., Simnett, G. M., Strong, K. T., Bentley, R. D., Bornmann, P. L., Bruner, M. E., Cargill, P. J., Crannell, C. J., Doyle, J. G., Hyder, C. L., Kopp, R. A., Lemen, J. R., Martin, S. F., Pallavicini, R., Peres, G., Serio, S., Sylwester, J., and Veck, N. J. 1986, Energetic Phenomena on the Sun, eds. B. E. Woodgate and M. R. Kundu (NASA Conference Procedings 2439), chapter 5. 